NOTICE: this is the author's version of a work that was accepted for publication in International Journal of Mechanical Sciences. Changes resulting from the publishing process, such as peer review, editing, corrections, structural formatting, and other quality control mechanisms may not be reflected in this document. Changes may have been made to this work since it was submitted for publication. A definitive version was subsequently published in International Journal of Mechanical Sciences, Vol. 74 (2013). DOI: 10.1016/j.jjmecsci.2013.05.002 


\title{
Improved Damage Identification in Bridge Structures Subject to Moving Loads: Numerical and Experimental Studies
}

\author{
Jun $\mathrm{Li}^{\mathrm{a}}{ }^{\mathrm{a} b}$, S.S. Law ${ }^{\mathrm{a}}$ and Hong Hao ${ }^{\mathrm{b}}$ \\ ${ }^{a}$ Department of Civil and Environmental Engineering, The Hong Kong Polytechnic University, \\ Hunghom, Kowloon, Hong Kong, People's Republic of China. \\ ${ }^{b}$ School of Civil and Resource Engineering, The University of Western Australia, \\ 35 Stirling Highway, Crawley, WA 6009, Australia.
}

\begin{abstract}
This paper proposes a damage identification approach in bridge structures under moving vehicular loads without knowledge of the vehicle properties and the time-histories of moving interaction forces. The dynamic response reconstruction technique in wavelet domain is developed for a structure subject to moving vehicular loads. The transmissibility matrix between two sets of time-domain response vectors from the structure is formulated using the unit impulse response function in the wavelet domain with the moving loads at different locations. Measured acceleration responses of the structure in the damaged state are required for the identification, and the damage identification procedure is conducted without knowledge of the time-histories of the moving loads. A dynamic response sensitivity-based method is used for the structural damage identification, and local damage is modeled as a change in the elemental stiffness factors. The adaptive Tikhonov regularization technique is adopted to improve the identification results when noise effect is included in the measurements. Numerical studies on a three-dimensional box-section girder are conducted to illustrate the effectiveness and performance of the proposed approach, and the simulated damage can be effectively identified even with $10 \%$ noise in the measurements. The proposed method is also found capable to identify the damage zone with an approximate estimation of the damage extent when under the influence of initial model errors of the structure. Experimental studies on a Tee-section prestressed concrete beam subject to a moving vehicle are preformed to validate the proposed approach. Identification results from the experimental test data show that the damage locations can be identified with a reasonable estimate of the damage extent.
\end{abstract}

\footnotetext{
Keywords: damage identification; response reconstruction; transmissibility; unit impulse response function; wavelet; moving vehicular loads; bridge structure; dynamics;

Email addresses: jun.li@uwa.edu.au; LI.Jun@connect.polyu.hk (Jun Li); cesslaw@polyu.edu.hk (S.S. Law); hong.hao@uwa.edu.au (Hong Hao)
} 


\section{Introduction}

The interest to monitor a structure for detecting local damage at an early stage is prevailing throughout the civil, mechanical and aerospace engineering communities. Significant work has been done in the detection of local damage in structures using changes in structural dynamic properties, such as natural frequency, mode shape, mode shape curvature and flexibility, etc. An important issue in this research area is to detect the local damage using measured responses from the structure under moving vehicular loads which serve as excitations to the structure. Lee et al. [1] presented a method for damage estimation of a simple bridge structure using vibration data caused by the traffic loads. The operational modal properties are identified basing on which the damage assessment is conducted using the neural network technique. Farrar and James [2] identified the modal properties of a bridge structure by curve-fitting the cross-correlation functions between two response measurements using the traffic excitation as the vibration source. A comparison between the identified results with those from standard forced vibration methods show that a maximum discrepancy of $3.63 \%$ in the natural frequency exists. This phenomenon is also found in field measurements with vehicles moving on top of the bridge deck. Also heavy vehicle has been reported to reduce the system stiffness while light vehicle increases the stiffness [3]. When these identified modal parameters are used to detect local damage in the structure directly, errors would be introduced due to the ignoring of the bridge-vehicle system interaction.

Traffic excitations usually occur together with other ambient excitation sources, such as earth micro-tremor, wind loading and temperature effect for bridge structures in real situations. The response due to the moving vehicular loads is generally far larger than that under ambient vibrations especially for short- and medium-span concrete bridge decks. Therefore damage identification could also be conducted in the time domain using measured dynamic responses directly instead of the modal information in the frequency domain. Majumder and Manohar [4] developed a time-domain approach to detect damages in a beam using vibration data under the passage of a moving oscillator. The study combines finite element modeling for the vehicle-bridge system with a time-domain formulation to detect changes in the structural parameters. The structural properties and motion of the moving vehicle are assumed to be known. Park et al. [5] proposed a method to identify the distribution of stiffness reductions in a damaged reinforced concrete slab bridge under 
moving loads by using a modified bivariate Gaussian distribution function. The information of moving loads is assumed available in this study. A method for simultaneous identification of moving masses and structural local damage from measured responses has been presented [6]. The masses and damage extents are taken as the optimization variables. However, the mass model may not accurately represent the moving vehicle and the bridge-vehicle interaction effect. In practical applications, the properties of the moving vehicle and the road surface roughness are not easy to obtain and thus they are usually assumed as unknown. The interaction forces induced by the moving vehicle should be treated as unknown moving load time-histories.

It is desirable to conduct the system identification based only on the system output (vibration responses of the bridge) because the system input (traffic excitations) is difficult to measure. With the aid of high computation capacity of digital computers, it is possible to analyze the bridge-vehicle interaction problem with more sophisticated bridge and vehicle models [7]. Zhu and Law [8] proposed a method for simultaneous identification of the time-histories of interaction forces and structural damage iteratively using a two-step identification procedure. Later, the structural condition assessment problem is studied in a three-span box-section concrete bridge deck subject to a three-dimensional moving vehicle by identifying the time-histories of the interaction forces and system parameters simultaneously in an iterative manner [9]. The effect of bridge-vehicle system interaction and road surface roughness profile are implicitly taken into account by identifying the moving interaction forces using measurements from the bridge structure. It is found that a sufficient number of sensors may be required to make sure that the identification equation for simultaneous identification of the interaction forces and system parameters is over-determined. It is also noted that the accuracy of the identified moving loads may have a large influence on the identification accuracy of the structural damage.

In the frequency domain analysis, Fast Fourier Transform (FFT) is a valuable tool for the analysis of vibration responses with the time domain signals transformed into frequency spectrum. However, leakage, end effects and aliasing occur in the FFT process. Filtering, windowing and ensemble-averaging techniques are often employed to alleviate these deficiencies with some success. Nevertheless, these errors in the FFT process still exist which may lead to a reduced accuracy in the subsequent analysis. More importantly, the basis functions associated with each frequency component in the Fourier-transformed domain span the entire measured time interval, 
hence making different signals indistinguishable as long as their spectral density is the same. Another disadvantage of Fourier analysis is that the frequency information can only be extracted from the complete duration of a signal record. When there is a local oscillation representing a particular feature at some point in the time history of a signal, it will contribute to the calculated Fourier spectrum but its information on the time axis will be lost [10]. Such disadvantage can be overcome in the wavelet analysis which is an alternative significant tool in signal analysis.

The generalized transmissibility matrix for a multi-degrees-of-freedom (MDOFs) system in frequency domain has been proposed by Ribeiro et al. [11]. The transmissibility matrix between two sets of response vectors could be used for the structural response reconstruction [12]. This response reconstruction is explored in the wavelet domain [13] to avoid the above-mentioned errors in the FFT process. Studies show that the wavelet approach is more accurate than the frequency approach and it is not subject to both the sampling duration and sampling rate. It should be noticed that the excitations involved in the above works are general forces at some specific locations. It has also been reported [14] that the deconvolution using the wavelet domain method for system identification show advantages over that using the frequency or time domain method since the wavelet domain method does not exhibit the rank-deficiency or ill conditioning in the computation of pseudo-inverse of a matrix, and this is a key attribute of the wavelet analysis methods.

The above literature review shows that existing methods need to identify the vehicle-bridge interaction loads from measured responses of the structure and the accuracy of damage identification results depends on the accuracy of the identified moving loads. This paper proposes a damage identification approach where knowledge of the moving vehicular loads is not required in the damage detection process. The dynamic response reconstruction technique in wavelet domain [13] is developed for a structure subject to moving vehicular loads. The transmissibility matrix between two sets of time-domain response vectors of the structure is formulated using the unit impulse response function in the wavelet domain with the moving loads at different locations. Measured acceleration responses from the structure in the damaged state are used for the damage detection. A dynamic response sensitivity-based method is used for the structural damage identification, and local damage is modeled as a change in the elemental stiffness factors. The adaptive Tikhonov regularization technique is adopted to improve the identification results, particularly, when noise effect is included in the measurements. Numerical studies on a 
three-dimensional box-section girder are conducted to illustrate the effectiveness and performance of the proposed approach, and the simulated damage can be effectively identified even with $10 \%$ noise in the measurements. The proposed method is also found to identify the damage zone with an approximate estimation of the damage extent when under the influence of initial model errors of the structure. Finally, experimental studies on a Tee-section prestressed concrete beam subject to a moving vehicle are preformed to verify the validity of the proposed approach.

\section{Response reconstruction in a structure subject to moving vehicular loads}

\subsection{Dynamic response of a structure under moving vehicular loads}

The governing equation of motion of a damped structural system with $N$ degrees-of-freedom (DOFs) subject to moving vehicular loads can be written as,

$$
[M]\{\ddot{x}(t)\}+[C]\{\dot{x}(t)\}+[K]\{x(t)\}=\left\{R_{l}(t)\right\}\left\{P_{\text {int }}(t)\right\}
$$

where $M, C$ and $K$ are the $N \times N$ mass, damping and stiffness matrices of the structure respectively; $\ddot{x}, \dot{x}$ and $x$ are respectively the acceleration, velocity and displacement response vectors of the structure; $\left\{P_{\text {int }}(t)\right\}$ is the bridge-vehicle interaction force vector acting on the bridge structure. $\left\{R_{l}(t)\right\}\left\{P_{\text {int }}(t)\right\}$ is the equivalent nodal load vector applied on the structure at location $l$ at time constant $t$ with the mapping vector $R_{l}(t)$. The vector $R_{l}(t)$ is time-varying and it can be represented by the shape function for computing the equivalent nodal loads [15]. Rayleigh damping $[C]=a_{1}[M]+a_{2}[K]$ is assumed, where $a_{1}$ and $a_{2}$ are the Rayleigh damping coefficients. It is noted the other damping model may be assumed for the proposed method. The dynamic responses of the structure can be obtained from Equation (1) using the Newmark- $\beta$ method [16].

\subsection{Unit impulse response function in wavelet domain}

The unit impulse response (UIR) (or the Markov parameters) is the response function of the system under the input of a unit pulse at a specific location, and it is an intrinsic function of the structural system. Traditionally, FFT is used to extract the impulse response data by an inverse FFT of the frequency response curves obtained from measured input and output [17]. It has been reported [18] that the impulse response data is extracted via the wavelet transform from known 
measured responses and input excitation information to avoid errors in the Fourier transformation process of both the input and output signals. Recently, the wavelet-based unit impulse response function with the force vector applied at a specific DOF has been derived analytically from the general system equation of motion and it will be introduced briefly below [19]. It will be extended in next Section for a structure subject to moving loads.

The equation of motion of a N DOFs damped structural system under the unit impulse excitation can be written as

$$
[M]\{\ddot{x}(t)\}+[C]\{\dot{x}(t)\}+[K]\{x(t)\}=D \delta(t)
$$

where $D$ is the mapping vector with zero entries except with unity at the DOF corresponding to the force excitation location, and $\delta(t)$ is the Dirac delta function. The impulse response function can be represented by a system in free vibration state with some specific initial conditions as

$$
\left\{\begin{array}{c}
{[M] \ddot{h}(t)+[C] \dot{h}(t)+[K] h(t)=0} \\
h(0)=0, \quad \dot{h}(0)=M^{-1} D
\end{array}\right.
$$

where $h, \dot{h}$ and $\ddot{h}$ are the unit impulse displacement, velocity and acceleration vectors, respectively. Assuming that the system is in static equilibrium initially, the unit impulse response function can be computed from the equation of motion using the Newmark- $\beta$ method.

When the structural system is under general excitation $f(t)$ with zero initial conditions, the acceleration response $\ddot{x}_{s}(t)$ from sensor location $s$ at time instant $t$ is

$$
\ddot{x}_{s}(t)=\int_{0}^{t} \ddot{h}_{s}(t-\tau) f(\tau) d \tau
$$

in which, $\ddot{h}_{s}(t)$ is the unit impulse response function at sensor location $s$. Equation (4) represents the input-output relationship of the dynamic structural system under the input force $f(t)$ at a specific location.

\subsection{Unit impulse response function in wavelet domain subject to moving loads}

It should be noticed that the mapping vector $\left\{R_{l}(t)\right\}$ in Equation (1) is time-varying when the structure is subject to moving vehicular loads. The impulse response function with the moving loads at different locations is derived in the following paragraphs to formulate the input-output 
relationship for the structure when subject to the interaction forces $\left\{P_{\text {int }}(t)\right\}$.

The equation of motion of the damped structural system under the unit impulse interaction force at location $l$ at a specific time instant $t$ is,

$$
[M]\{\ddot{x}(t)\}+[C]\{\dot{x}(t)\}+[K]\{x(t)\}=\left\{R_{l}(t)\right\} \delta(t)
$$

where, $R_{l}(t)$ denotes the shape function mapping the interaction force at location $l$ at time instant $t$ to the associated DOFs of the structure. Similar to the solution of Equation (2), the impulse response function with the moving loads at location $l$ can be obtained using the Newmark- $\beta$ method by solving the following equation of motion with some specific initial conditions,

$$
\left\{\begin{array}{c}
{[M] \ddot{h}_{l}(t)+[C] \dot{h}_{l}(t)+[K] h_{l}(t)=0} \\
h_{l}(0)=0, \quad \dot{h}_{l}(0)=M^{-1} R_{l}(t)
\end{array}\right.
$$

where, $h_{l}, \dot{h}_{l}$ and $\ddot{h_{l}}$ are the unit impulse displacement, velocity and acceleration vectors with the moving loads at location $l$, respectively.

When the structural system is subject to the moving load $P_{\text {int }}(t)$ with zero initial conditions, the acceleration response $\ddot{x}_{s}(t)$ from sensor location $s$ at time instant $t$ can be obtained as,

$$
\ddot{x}_{s}(t)=\int_{0}^{t} \ddot{h}_{s, l_{t}}(t-\tau) P_{\mathrm{int}}(\tau) d \tau
$$

in which, $\ddot{h}_{s, l_{\tau}}(t)$ is the unit impulse response function with the moving loads at location $l_{\tau}$ for sensor location $s$. It is noted that $\ddot{h}_{s, l_{\tau}}(t)$ can be obtained from Equation (6) with the moving loads placed at different locations one time step at a time. It should be noted that the formulation of Equation (7) is different from that of Equation (4) since the impulse response function with the moving loads at different locations will be used in Equation (7) rather than the same impulse response function with the input force at a specific location for Equation (4). The vectors $\ddot{h}_{s, l_{\tau}}(t-\tau)$ and $P_{\text {int }}(\tau)$ can be expanded in terms of the discrete wavelet transform (DWT) as [10],

$$
\begin{gathered}
\ddot{h}_{s, l_{\tau}}(t-\tau)=h_{s, 0}^{D W T}+\sum_{j} \sum_{k} h_{s, 2^{j}+k}^{D W T} \psi\left(2^{j} \tau-k\right) \\
P_{\mathrm{int}}(\tau)=P_{0}^{D W T}+\sum_{j} \sum_{k} P_{2^{j}+k}^{D W T} \psi\left(2^{j} \tau-k\right)
\end{gathered}
$$


where $\psi\left(2^{j} \tau-k\right)$ is the wavelet basis function, $h_{s, 2^{j}+k}^{D W T}$ and $P_{2^{j}+k}^{D W T}$ are the wavelet transform coefficients for the impulse response function and moving force vectors respectively. Substituting Equations (8) and (9) into the convolution integral in Equation (7), and using the orthogonal conditions of the wavelet basis functions [20] as follows,

$$
\begin{gathered}
\int_{0}^{t} \psi\left(2^{j} \tau-k\right) d \tau=0 \\
\int_{0}^{t} \psi\left(2^{j} \tau-k\right) \psi\left(2^{r} \tau-s\right) d \tau=\left\{\begin{array}{cc}
1 / 2^{j} & \text { when } r=j \text { and } s=k \\
0 & \text { otherwise }
\end{array}\right.
\end{gathered}
$$

the following formula can then be derived as

$$
\ddot{x}_{s}(t)=\ddot{h}_{s}^{D W T}(t) P_{\mathrm{int}}^{D W T}
$$

in which, $\ddot{h}_{s}^{D W T}(t)$ and $P_{\mathrm{int}}^{D W T}$ are the discrete wavelet transforms of $\ddot{h}_{s, l_{\tau}}(t-\tau)$ and $P_{\text {int }}(\tau)$, respectively and they are given as,

$$
\begin{gathered}
P_{\mathrm{int}}^{D W T}=\left[\begin{array}{llll}
P_{0}^{D W T} & P_{1}^{D W T} & \cdots & P_{2^{j}+k}^{D W T}
\end{array}\right]^{T} \\
\ddot{h}_{s}^{D W T}(t)=\left[\begin{array}{llll}
\ddot{h}_{s, 0}^{D W T}(t) & \ddot{h}_{s, 1}^{D W T}(t) & \cdots & \ddot{h}_{s, 2^{j}+k}^{D W T}(t) / 2^{j}
\end{array}\right]
\end{gathered}
$$

For the entire time history data, for example, $\ddot{x}_{s}=\left[\begin{array}{llll}\ddot{x}_{s}\left(t_{1}\right) & \ddot{x}_{s}\left(t_{2}\right) & \cdots & \ddot{x}_{s}\left(t_{n}\right)\end{array}\right]^{T}$, the system input-output relationship for the structure subject to moving loads can be expressed as,

$$
\ddot{x}_{s(n \times 1)}=\ddot{h}_{s}^{D W T}{ }_{(n \times r u)} P_{\mathrm{int}}^{D W T}{ }_{(r u \times 1)}
$$

in which,

$$
\ddot{h}_{s}^{D W T}=\left[\begin{array}{c}
\ddot{h}_{s}^{D W T}\left(t_{1}\right) \\
\ddot{h}_{s}^{D W T}\left(t_{2}\right) \\
\vdots \\
\ddot{h}_{s}^{D W T}\left(t_{n}\right)
\end{array}\right]
$$

where $n, r$ and $u$ are the number of sampled data in the response, the number of moving loads and the number of wavelet coefficients in the discrete wavelet transform, respectively.

\subsection{Response reconstruction in a structure subject to moving loads}

The measured responses from the structure subject to moving loads are divided into two sets, noted as the First-set response vector $\ddot{x}_{1}(t)$ and the Second-set response vector $\ddot{x}_{2}(t)$ respectively. 
They are represented in the wavelet domain from Equation (13) as follows,

$$
\left\{\begin{array}{l}
\ddot{x}_{1}(t)_{(m n \times 1)}=\ddot{h}_{1}^{D W T}{ }_{(m n \times r u)} P_{\mathrm{int}}^{D W T}{ }_{(r u \times 1)} \\
\ddot{x}_{2}(t)_{(q n \times 1)}=\ddot{h}_{2}^{D W T}{ }_{(q n \times r u)} P_{\mathrm{int}}^{D W T}{ }_{(r u \times 1)}
\end{array}\right.
$$

in which, $m$ and $q$ are the number of measurements in the First-set response vector and the Second-set response vector, respectively.

When the number of measurements in the First-set response vector is at least equal or larger than the number of moving loads on the structure, the pseudo-inverse $\left(h_{1}^{D W T}\right)^{+}$exists [21] and the following equation can be obtained from the first row of Equation (14)

$$
P_{\mathrm{int}}^{D W T}=\left(\ddot{h}_{1}^{D W T}\right)^{+} \ddot{x}_{1}(t)
$$

Substituting Equation (15) into the second row of Equation (14), we have

$$
\ddot{x}_{2 r}(t)=T_{12} \ddot{x}_{1}(t)
$$

where,

$$
T_{12}=\ddot{h}_{2}^{D W T}\left(\ddot{h}_{1}^{D W T}\right)^{+}
$$

The Second-set response vector $\ddot{x}_{2 r}(t)$ can be reconstructed from the First-set response vector $\ddot{x}_{1}(t)$ of the structure from Equation (16). Moreover, Equation (17) defines the transmissibility matrix in wavelet domain between two sets of time-domain response vectors from the structure, and the presented response reconstruction technique for a bridge structure subject to moving loads can be applied for the structural damage identification in next section.

\section{Structural damage identification}

In some existing condition assessment approaches where an initial analytical finite element model of the structure is needed, the parametric model updating method for damage identification is popular because it keeps the structural connectivity and the physical meaning of the updated stiffness matrix is clear. The initial structural finite element model is updated to match the predicted and measured vibration properties or vibration responses as closely as possible. In this study, a sensitivity-based finite element model updating method is used for structural damage identification. The damage is assumed only related to a stiffness reduction such as a change in the elastic modulus 
of a specific element. The mass matrix is assumed to be unchanged before and after the damage. The elemental stiffness factors in the initial intact structural finite element model are iteratively updated to minimize the difference vector $\{\Delta \ddot{x}\}$ between the reconstructed acceleration responses $\ddot{x}_{2 r}(t)$ and the measured acceleration responses $\ddot{x}_{2 m}(t)$ from the damaged structure.

\subsection{Damage model}

A linear damage model is adopted in this study, i.e., the initially linear-elastic structure is assumed to remain linear-elastic after the occurrence of small local damage. The system stiffness matrix $K_{d}$ of the damaged structure can be expressed as,

$$
K_{d}=\sum_{i=1}^{n} \alpha_{i} K_{i}=\sum_{i=1}^{n}\left(1+\Delta \alpha_{i}\right) K_{i}
$$

where, $K_{i}, \alpha_{i}$ are the $i$ th elemental stiffness matrix in the intact state and the $i$ th elemental stiffness factor in the damage state, respectively. Therefore, $\Delta \alpha_{i}$ represents the extent of stiffness reduction of the $i$ th element with $0.0 \leq \alpha_{i} \leq 1.0$.

\subsection{Damage detection algorithm}

The objective function of the damage detection algorithm is defined as the difference between two sets of response vectors

$$
f_{o b j}=\left\|\ddot{x}_{2 m}(t)-\ddot{x}_{2 r}(t)\right\|_{2}
$$

where, $\ddot{x}_{2 m}(t)$ is the measured Second-set response vector from the damaged structure subject to moving loads. $\ddot{x}_{2 r}(t)$ is the reconstructed Second-set response vector from Equation (16) with the measured First-set response vector $\ddot{x}_{1}(t)$ in the damaged state. When two or more responses are included in Equation (19), they will be assembled in a vector of time response data of one sensor following by those from another sensor. The wavelet transform coefficients of the measured and reconstruction responses can also be included in Equation (19) as the objective function. However, Equation (19) gives a clear physical meaning with matching of the measured and reconstruction 
responses as closely as possible in the optimized results. The transmissibility matrix $T_{12}$ in Equation (16) is obtained by using the impulse response function matrix from Equation (13). The vector $\alpha$ of structural elemental stiffness factors is then iteratively updated by minimizing the objective function in Equation (19) such that the reconstructed response vector $\ddot{x}_{2 r}(t)$ can match the measured response vector $\ddot{x}_{2 m}(t)$ well.

The dynamic response sensitivity-based model updating method [22] without considering the second- and higher-order effects is adopted here with

$$
[S]\{\Delta \alpha\}=\{\Delta \ddot{x}\}=\left\{\ddot{x}_{2 m}\right\}-\left\{\ddot{x}_{2 r}\right\}
$$

where, $\Delta \alpha$ is the perturbation of the vector of structural elemental stiffness factors, $[S]$ is the sensitivity matrix of the response $\ddot{x}_{2 r}(t)$ with respect to the structural elemental stiffness factors. The objective function in Equation (19) is an implicit function with respect to structural elemental stiffness factors. It has been verified that the numerical sensitivity matrix can also be used for model updating effectively [23], and thus the sensitivity matrix $[S]$ is obtained using numerical finite difference method [24]. It is noted that the number of equations $q \times n$ should be larger than the number of unknown elemental stiffness parameters to make sure that the identification in Equation (20) is over-determined. The iterative updating method requires the sensitivity matrix $[S]$ to be calculated in every iteration. The sensitivity matrix is a rectangular matrix of order $q \times U$, where $q$ and $U$ are the number of target responses in Equation (19) and system parameters to be identified, respectively

$$
[S]=\left[S_{1}, S_{2}, \cdots, S_{U}\right]=\left[\frac{\partial \ddot{x}_{2 r}(t)}{\partial P_{j=1,2, \cdots, U}}\right]
$$

$S_{j}(j=1,2, \cdots, U)$ is the sensitivity of the target responses to a certain change in parameter $P_{j}$. Elements of the sensitivity matrix can be calculated numerically using, for example, the forward finite difference approach [23]

$$
S_{j}=\frac{\ddot{x}_{2 r}\left(P_{j}+\Delta P_{j}\right)-\ddot{x}_{2 r}\left(P_{j}\right)}{\Delta P_{j}}
$$


where $\ddot{x}_{2 r}\left(P_{j}\right)$ is the reconstructed second-set response at the current state of the parameter $P_{j}$, while $\ddot{x}_{2 r}\left(P_{j}+\Delta P_{j}\right)$ is the reconstructed response when the parameter $P_{j}$ is increased by an increment $\Delta P_{j}$.

\subsection{Adaptive Tikhonov regularization}

The adaptive Tikhonov regularization method [25] has been proposed to improve the model updating results by categorizing all the structural elements into two groups of possible damaged elements and intact elements from results obtained in the previous iteration. The perturbation of elemental stiffness reduction factors of the possible damaged elements in each iteration is limited to a small range and the reduction factors of other elements are restrained close to zeros as

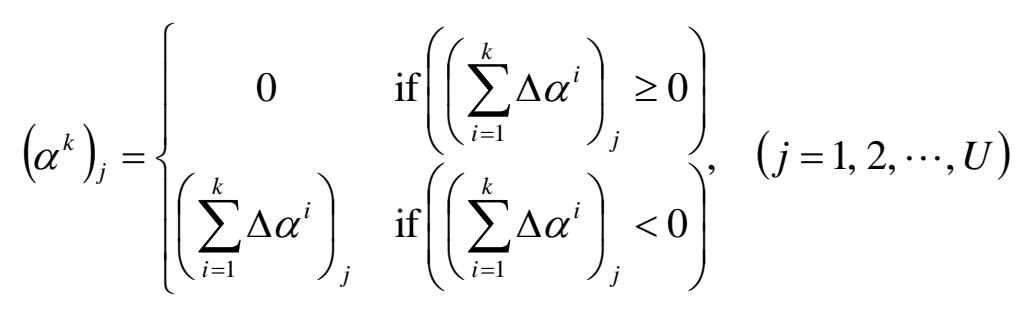

where the subscript $j$ denotes $j$ th element of the vector and $\left(\alpha^{k}\right)_{j}$ denotes the parameter change in the $j$ th element. $\left(\sum_{i=1}^{k} \Delta \alpha^{i}\right)_{j}<0$ indicates that the accumulated change in the $j$ th element is a decrease in the stiffness of the element.

It has been shown that the adaptive Tikhonov regularization has obvious advantages over the traditional Tikhonov regularization with less false positives and false negatives especially when relatively high noise level exists in the measurements. On the other hand, the adaptive Tikhonov regularization can give results without divergence but with a slower convergence speed. The adaptive Tikhonov regularization technique is used in this study to obtain the solution vector $\Delta \alpha$ from Equation (20). More details and implementation of the adaptive Tikhonov regularization are referred to [25].

\subsection{Iterative damage detection procedure}

Acceleration measurements from the damaged structure under the passage of the moving loads 
will be used to identify structural elemental stiffness factors $\alpha_{i}$ iteratively. Initially it is assumed that each elemental stiffness factor of the analytical structural finite element model is equal to unity. It should be noted that the travelling path and velocity of the moving loads, i.e. the locations, are assumed to be known in the process. An updated finite element model is assumed to be available as a reference model for the following iterative procedure of damage identification.

Step 1: Measure the dynamic acceleration responses at the First-set $\left\{\ddot{x}_{1 m}(t)\right\}$ and Second-set $\left\{\ddot{x}_{2 m}(t)\right\}$ measurement locations from the damaged structure subject to the moving loads.

Step 2: Compute the unit impulse response function matrices $\ddot{h}_{1}^{D W T}$ and $\ddot{h}_{2}^{D W T}$ in Equation (14) for the First-set and Second-set measurement DOFs respectively from the analytical finite element model of the structure. Calculate matrix $T_{12}$ in Equation (17) and the reconstructed Second-set response vector $\left\{\ddot{x}_{2 r}(t)\right\}$ is obtained from Equation (16).

Step 3: The vector of response difference $\{\Delta \ddot{x}\}$ is computed between the Second-set measured response vector $\left\{\ddot{x}_{2 m}(t)\right\}$ in Step 1 and the reconstructed Second-set response vector $\left\{\ddot{x}_{2 r}(t)\right\}$ in Step 2. The sensitivity matrix $[S]$ of the response $\ddot{x}_{2 r}(t)$ with respect to structural elemental stiffness factors is obtained using the numerical finite difference method.

Step 4: Obtain the perturbation vector of structural elemental stiffness factors $\{\Delta \alpha\}$ from Equation (20) with the adaptive Tikhonov regularization technique described in Section 3.3.

Step 5: The vector of structural elemental stiffness factors is iteratively updated with $\alpha_{i+1}=\alpha_{i}+\Delta \alpha$ for the next iteration. Repeat Steps 2 to 4 until the following convergence criterion is satisfied.

$$
\frac{\left\|\alpha_{i+1}-\alpha_{i}\right\|_{2}}{\left\|\alpha_{i}\right\|_{2}} \leq \text { Tolerance }
$$

where $i$ denotes the $i$ th iteration. The tolerance value is taken as $1.0 \times 10^{-4}$ in this study. In the above-mentioned iterative scheme for damage identification, it should be noticed that: (a) the properties of the moving vehicle and the time-histories of the moving loads on the bridge 
structure are not required to be identified; (b) the locations of the moving loads are assumed to be known.

\section{Numerical studies}

Numerical studies on a simply-supported box-section girder bridge structure are conducted to illustrate the accuracy and effectiveness of the proposed structural damage identification approach. The total length of the box-section bridge deck is $30 \mathrm{~m}$. The plan view and cross-section of the bridge deck model are shown in Figures 1(a) and 1(b), respectively. The Young's modulus and mass density are respectively $2.6 \times 10^{4} \mathrm{MPa}$ and $2500 \mathrm{~kg} / \mathrm{m}^{3}$.

The finite element model of the bridge deck consists of 66 nodes and 60 flat shell elements [26] with six DOFs at each node. The numberings of nodes and elements of the finite element model are shown in Figure 1. The structural system has 396 DOFs in total. The bridge deck is simply-supported at nodes 5, 6, 65 and 66 at two ends of the deck, and the translational restraints at the supports are represented by a large stiffness of $3 \times 10^{9} \mathrm{kN} / \mathrm{m}$. The first ten intact structural natural frequencies are from 4.44 to $21.61 \mathrm{~Hz}$. Rayleigh damping is assumed in this study and the damping ratios for the first two modes are taken as $\xi=0.012$.

In engineering applications, moving loads induced by the passage of a vehicle is often considered as excitations to the bridge structures. The moving load has been represented very often as a multi-sine wave moving force in many studies [8, 27] for an easier and simpler structural analysis of the bridge-vehicle system. The first numerical study has the box-section girder bridge subject to a single multi-sine wave moving force. The second example has the girder structure under the passage of a two-axle three-dimensional vehicle which represents the more realistic moving vehicle model. The road surface roughness effect will also be included in the bridge-vehicle system analysis.

\subsection{Example 1: The bridge deck subject to a single moving force}

Damage is introduced in the box-section bridge deck as a reduction of elastic modulus in several elements. In this study, $10 \%$ damage is simulated in both the $28^{\text {th }}$ and $29^{\text {th }}$ elements at mid-span of the deck as shown in Figure 1. The moving force is represented as, 


$$
P(t)=160000(1+0.1 \sin (10 \pi t)+0.05 \sin (30 \pi t)) N
$$

Damage identification is performed with the moving force crossing the bridge along the centerline of the deck as shown in Figure 1(a). The force vector acting at an arbitrary location on a shell element of the bridge deck is transformed into nodal loads using the Hermite interpolation function [28]. Six sensors are assumed distributed on top of the deck to measure the acceleration responses from the damaged structure. The measurements are divided into two sets of responses and they are shown in Table 1. The number of measurements in the First-set response vector is two and it is greater than the number of moving force. The sensors locations are such selected to have larger response which usually associated with a larger signal to noise ratio. The velocity of the moving force is $20 \mathrm{~m} / \mathrm{s}$ and the sampling rate is $100 \mathrm{~Hz}$. The acceleration response data of the damaged structure calculated from Equation (1) serves as the simulated "measured" responses, and the first 3 seconds of response are used except otherwise stated.

\subsubsection{Forward response reconstruction in wavelet domain}

The accuracy of response reconstruction in the structure subject to moving loads will be examined. The simulated local damages are introduced in the structure and the responses are obtained at the First-set and Second-set sensor locations in the damaged state. The reconstructed Second-set response vector is obtained from Equation (16) and is compared with the analytical Second-set response which is considered as the true response. In this study, Daubechies 8-coefficient wavelet is chosen as the basis functions in the DWT due to its orthogonality properties and fairly smooth interpolation nature [18]. It should be noticed that no noise is added to the simulated "measured" data in this study. Results of forward response reconstruction are shown in Figure 2. Figures 2(a), 2(c), 2(e) and 2(g) show the true and reconstructed responses at those sensor locations in the Second-set response vector, respectively. It can be found that these two responses are overlapping indicating that the response reconstruction process is very accurate.

The relative error between the true and reconstructed responses in the time domain is defined as,

$$
R E=\frac{\left\|\ddot{x}_{\text {true }}(t)-\ddot{x}_{u r}(t)\right\|_{2}}{\left\|\ddot{x}_{\text {true }}(t)\right\|_{2}} \times 100(\%)
$$


in which, $\ddot{x}_{\text {true }}(t)$ and $\ddot{x}_{u r}(t)$ are the true and reconstructed response vectors in the time domain, respectively.

The difference vectors $\left(\ddot{x}_{\text {true }}(t)-\ddot{x}_{u r}(t)\right)$ between the true and reconstructed responses of the sensors in the Second-set response vector are shown in Figures 2(b), 2(d), 2(f) and 2(h). The relative errors for these four sensors are $5.97 \times 10^{-12}, 8.47 \times 10^{-12}, 7.47 \times 10^{-12}$ and $3.48 \times 10^{-12}$, respectively indicating that the proposed method for dynamic response reconstruction in the structure under the passage of the moving force is very accurate. It may also be noted that different sensor placement configurations in the First-set and Second-set response vectors may give different accuracies in the response reconstruction process.

\subsubsection{Results of damage identification}

The iterative procedure described in Section 3.4 is followed to obtain an updated set of elemental stiffness factors. To simulate the effect of measurement noise, a normally distributed random noise with zero mean and unit standard deviation is added to the calculated dynamic response as,

$$
\ddot{x}_{n}=\ddot{x}_{c a l}+E_{p} N_{\text {oise }} s t d\left(\ddot{x}_{c a l}\right)
$$

where $\ddot{x}_{n}$ and $\ddot{x}_{c a l}$ are the simulated measurements with noise effect and the original calculated response, respectively; $E_{p}$ is the noise level; $N_{\text {oise }}$ is a standard normal distribution vector with zero mean and unit standard deviation and $\operatorname{std}\left(\ddot{x}_{c a l}\right)$ denotes the standard deviation of the original calculated response. $10 \%$ noise effect is included in the acceleration measurements for this study.

Acceleration measurements with and without noise effect are used for damage identification, respectively. Table 2 gives the associated information on convergence of the iterative procedure. The required iterations and error of convergence calculated from Equation (24) are listed. It should be noticed that approximately 1.5 hours are required for one iteration with a Intel Core 2 Quad 2.4G PC with $8 \mathrm{G}$ memory due to the large structural finite element model for the computation of responses and sensitivity matrix of the structure by the finite difference method.

The damage identification results are shown in Figure 3. For the noise-free case, the locations of the simulated damage can be identified accurately. The identified extents of local damage in $28^{\text {th }}$ 
and $29^{\text {th }}$ elements without noise effect are $9.997 \%$ and $9.999 \%$ respectively. They are very close to the true values indicating that the proposed approach for damage identification in the structure under moving loads is effective and can give very close damage values. For the case with $10 \%$ noise, the dynamic response data within the first 0.5 second and 1.5 to 2 seconds are used for the identification since the responses in these periods are much larger and could be less sensitive to the noise effect. Figure 3 shows that the damage can be identified effectively with $10 \%$ noise effect with $8.32 \%$ and $11.23 \%$ stiffness reductions in $28^{\text {th }}$ and $29^{\text {th }}$ elements respectively, and there is only a few very small false positives and false negatives in the other undamaged elements.

\subsection{Example 2: The bridge deck subject to a two-axle three-dimensional moving vehicle}

\subsubsection{Dynamic analysis of the bridge-vehicle system}

The vehicle is modelled according to the H20-44 truck in AASHTO [29] which is a two-axle three-dimensional vehicle model with seven DOFs as shown in Figure 4. The specific parameters of the vehicle are referred in [30], with a mass of $17000 \mathrm{~kg}$. The dynamic responses of the bridge structure are obtained by solving the coupled bridge-vehicle system equation of motion [31].

The two-axle three-dimensional vehicle crosses the bridge along the travelling path as shown in Figure 1(a). Seven sensors are assumed distributed on the deck in this case to measure the acceleration responses from the damaged bridge deck. The measurements are divided into two sets of responses and they are shown in Table 3. The number of measurements in the First-set response vector is equal to five and it is greater than the number of interaction forces induced by the moving vehicle which is four. The velocity of the moving force is $20 \mathrm{~m} / \mathrm{s}$ and the sampling rate is $100 \mathrm{~Hz}$. Class $C$ road surface roughness [32], corresponding to the average road pavement condition, is included in the bridge-vehicle system analysis. The calculated acceleration response of the damaged structure serves as the simulated "measured" response and data within the first 3 seconds are used except otherwise stated.

\subsubsection{Forward response reconstruction in wavelet domain}

The damage scenario in the girder structure is the same as for the last example, that is, $10 \%$ damage in both the $28^{\text {th }}$ and $29^{\text {th }}$ elements in the web of the bridge structure in the form of a reduction in the elastic modulus of these elements. The simulated local damages are introduced in 
the structure and responses are obtained at the First-set and Second-set sensor locations. The reconstructed Second-set response vector is obtained from Equation (16) and is compared with the "measured" true Second-set response. It should be noticed that no noise is added to the measurements. The comparisons of forward response reconstruction results are shown in Figure 5. Figures 5(a) and 5(c) show the true and reconstructed responses at the sensor locations in the Second-set response vector. The difference vectors $\left(\ddot{x}_{t r u e}(t)-\ddot{x}_{u r}(t)\right)$ between the true and reconstructed responses of these two sensors are shown in Figures 5(b) and 5(d). The relative errors are $1.74 \times 10^{-11}$ and $1.39 \times 10^{-11}$, respectively. These results indicated that the proposed response reconstruction method in the structure subject to moving vehicular loads is very accurate.

\subsubsection{Results of damage identification}

Damage identification is performed with the two-axle three-dimensional vehicle crossing the bridge along the travelling path as shown in Figure 1(a). 10\% noise effect is included in the acceleration measurements. The iterative procedure described in Section 3.4 is followed to conduct the damage identification.

Acceleration measurements with and without noise effect are used for the damage identification. Table 4 gives the associated information on convergence of the iterative procedure. The computation of matrix $T_{12}$ in this case becomes intensive since four interaction forces from the moving vehicle are applied on the bridge structure. It should be noticed that approximately 6 hours are required for one iteration with a Intel Core 2 Quad 2.4G PC with 8G memory in the bridge-vehicle system analysis and in the computation of the sensitivity matrix for the structure subject to moving loads.

Figure 6 shows the damage identification results. For the noise-free case, the damage locations and extents are identified accurately with $9.9996 \%$ and $9.9987 \%$ stiffness reductions in $28^{\text {th }}$ and $29^{\text {th }}$ element respectively. It indicates that the proposed approach for damage identification in the structure under moving vehicular loads is correct. For the case with $10 \%$ noise, response data in the first $0.8 \mathrm{~s}$ and 1.5 to $2.2 \mathrm{~s}$ are used for the identification since the responses in these periods are much larger and could be less sensitive to the noise effect. The damages can be identified effectively with $10.41 \%$ and $11.84 \%$ stiffness reduction in $28^{\text {th }}$ and $29^{\text {th }}$ elements respectively when $10 \%$ noise 
effect is included in the measurements with very small false positives and false negatives similar to the observations in Figure 3. In addition, it should be noticed that the sensor selections in the First-set and Second-set response vectors would influence the damage identification results especially for the case with noisy measurements. However, the issue on optimal placement of sensor in the First-set and Second-set response vectors is not examined in this study.

\subsection{Influence of initial model errors on the identification results}

The influence of initial model errors in the finite element model on the effectiveness and performance of the proposed approach is investigated in this Section. The initial model errors in the stiffness of elements and in the support stiffness are considered in this study. Other model error sources, such as uncertainties in the mesh and element type in the finite element analysis, mass matrix and temperature effect, etc. are not included.

Example 2 in Section 4.2 is adopted for this study. Three scenarios are defined in Table 5. $20 \%$ stiffness reductions are introduced in both the $28^{\text {th }}$ and $29^{\text {th }}$ elements of the deck. The elastic modulus of material in the finite elements is assumed to exhibit a normal random distribution [33] with a mean of $2.6 \times 10^{4} \mathrm{MPa}$ and a $5 \%$ coefficient of variation in Scenario 1 . The support stiffness has been over-estimated initially by $10 \%$ in Scenario 2 . Scenario 3 includes both types of initial model errors in the last two Scenarios.

The identification results of these three scenarios are shown in Figures 7(a), 7(b) and 7(c), respectively. In Scenario 1, the simulated damage in $28^{\text {th }}$ and $29^{\text {th }}$ elements and model errors in the stiffness of other undamaged elements are identified accurately for the noise-free case. For the case with $10 \%$ noise effect, the identified damages in $28^{\text {th }}$ and $29^{\text {th }}$ elements are $19.49 \%$ and $19.52 \%$, respectively. The model errors in stiffness may not be identified accurately in all elements and there are several large false positives and false negatives in the identification results.

In Scenario 2, the identification results in $28^{\text {th }}$ and $29^{\text {th }}$ elements are $14.48 \%$ and $12.41 \%$, and 21.90 and $11.26 \%$ for the case without and with $10 \%$ measurement noise, respectively. The adaptive Tikhonov regularization technique improves the identification results with no false negatives but with several false positives observed. The identified extents in $28^{\text {th }}$ and $29^{\text {th }}$ elements of Scenario 3 are $19.81 \%$ and $21.87 \%$, and $16.62 \%$ and $16.99 \%$ for the case without noise and with $10 \%$ noise, 
respectively. Several false positives and false negatives exist in the identification results. It may be concluded that the damage detection approach can identify the area with local damages when there is $5 \%$ random error in the initial finite element of the structure, and the damage extended can be approximately estimated.

\section{Experimental Verification}

Experimental studies on a Tee-section prestressed concrete beam are performed to validate the proposed damage identification approach with experimental test data. The plan view, cross section and dimensions of the beam are shown in Figure 8. The beam slab has a length of $5 \mathrm{~m}$ and a width of $0.65 \mathrm{~m}$. The total height of the beam is $0.415 \mathrm{~m}$. The initial Young's modulus and density are $2.6 \times 10^{4} \mathrm{MPa}$ and $2707.7 \mathrm{~kg} / \mathrm{m}^{3}$, respectively. Three prestressing tendons each of $99.8 \mathrm{~mm}^{2}$ area are included in the beam web with a total prestress force of $140 \mathrm{KN}$. The tensile strength of the tendons is $1949 \mathrm{~N} / \mathrm{mm}^{2}$. The cable profile is parabolic and locations of the prestress tendons at the beam ends and mid-span are shown in Figure 8. The cable duct is grouted after prestressing.

A vehicle is designed and fabricated as shown in Figure 9(a). The spacing of the front and rear axes is $0.8 \mathrm{~m}$ and the lateral spacing of two wheels is $0.4 \mathrm{~m}$. The total weight of the vehicle is $9.8 \mathrm{KN}$. Seven accelerometers are placed at the top of the beam to measure the acceleration under the moving vehicle loads, and six optical triggers are evenly distributed along the left side of the beam to measure the velocity of the moving vehicle, as shown Figure 9(b). The experimental set-up is shown schematically in Figure 10. An electric motor pulls the vehicle to move along the center line of the beam. A leading beam is included for initial acceleration of the vehicle while the vehicle decelerates on a tailing beam. The bridge is at rest initially, and the vehicle moves at approximately a constant speed of $0.4 \mathrm{~m} / \mathrm{s}$. The sampling rate is $1000 \mathrm{~Hz}$.

Figure 11 shows the flow chart of the experimental damage identification. The damage is introduced by applying the two-point static load of $180 \mathrm{kN}$ on the beam to introduce cracking damage into the structure, as shown in Figure 12. The observed cracks are mainly in the web elements in the middle of the span as shown in Figure 13. Measured data from the beam without prestress effect, with prestress effect and the beam with both prestress effect and damages are analyzed to obtain the modal information, and the identified natural frequencies and damping ratios 
are shown in Table 6. Comparing the identified frequencies from the states without and with prestress effect, it can be found that structural stiffness is increased due to the prestress effect with an increase in the natural frequencies. The identified frequencies from the damaged state are smaller than those from the initial state. This clearly indicates that damages have been introduced in the structure.

The beam with prestress is considered as the initial state and measured data from this state are used for the initial model updating to adjust the initial finite element model. The model updating is conducted based on a two-stage procedure. In the first-stage of initial model updating, the Young's modulus of slab and web of beam and the support stiffnesses are selected as parameters to be updated. The dimensions and mass density are measured in situ and they are not included as the updating parameters. The identified damping ratios of the beam are included in the initial finite element model. Identified modal information, e.g. natural frequencies and mode shapes, are used to perform the first-stage initial updating. Based on the updated results obtained above, the second-stage model updating is conducted based on the proposed approach. The equivalent elastic modulus of all the elements under the prestressing effect is selected in the second-stage of updating for damage identification. Sensor placement configuration in Table 7 is used, and the objective function of this round of model updating is to have the reconstructed responses in the Second-set match the measured ones as closely as possible. The iteration number is 3 and the regularization parameter of the final iteration is $3.5 \times 10^{-3}$.

Figure 14 shows the finite element model of the beam built with flat shell elements [26]. The model consisted of 90 elements and 114 nodes with 6 DOFs at a node. The model has 684 DOFs in total. Wheel tracks, moving direction of the vehicle, and the numbering of web elements are also shown in Figure 14. It is observed and understood that the damages are mainly and most likely occur in the web elements of the beam, and therefore only the web elements are included in the damage identification. Elements 1, 2, 17 and 18 are however not included as they are just next to the supports. Table 7 shows the sensor placement configuration for the experimental study. Five sensors are included in the First-set as there are four interaction forces from the moving vehicle. There are two sensors in the Second-set. Figure 15 shows the identified damages in the web elements from the first 2 seconds of measured responses with a resampled rate of $250 \mathrm{~Hz}$. It can be observed that the identified damages are mainly in the center span and the extents in the middle of 
the span with a maximum damage extent close to $12 \%$.

It is noted that there is no analytical model relating the crack damage in a prestressed concrete beam with its flexural stiffness, it is not possible to compare the identified damage extent with the true damage induced by static loads. However, Table 8 shows the observed crack locations and heights in the beam. There are 24 major cracks in total and they are mainly located in the six web elements from No.8 to No.13. The overall length of the crack zone is $3.6 \mathrm{~m}$. It is noted that the identified damage distribution in Figure 15 has a good agreement with the experimental crack pattern observed in Table 8 and Figure 13. The identified damages are mainly distributed in web elements from No.8 to No.13.

\section{Conclusions}

Existing methods for damage detection of bridges under moving vehicle loads assume that the properties of the vehicle are known or they require the simultaneous identification the interaction forces and structural damage. A structural damage identification approach is proposed for bridge structures under moving vehicular loads based on the dynamic response reconstruction without the need to identify the time-histories of the moving loads and the properties of moving vehicle and with improvements in the computation efficiency and accuracy. The relationship between two sets of time-domain response vectors from the structure is formulated using the unit impulse response function in the wavelet domain with the moving loads at different locations. A dynamic response sensitivity-based method is used for the structural damage identification. The adaptive Tikhonov regularization technique is adopted to improve the identification results, particularly, when noise effect is included in the measurements. Numerical studies on a three-dimensional box-section bridge deck subject to a single moving force and a two-axle three-dimensional vehicle are separately studied to validate the proposed approach. The influence of initial model errors on the damage identification results is investigated to illustrate the effectiveness and performance of the proposed approach and the simulated damage can be identified even with $10 \%$ noise in the measurements but with some false positives and false negatives. Experimental studies on a Tee-section prestressed concrete beam are conducted to validate the proposed approach with experimental test data. Two-point static loads are applied to introduce cracking damage in the beam. 
Measure acceleration response data from the initial and damage states are used to conduct initial model updating and damage identification, respectively. The identified damages are mainly in the middle of the span and there is a close agreement with the observed crack pattern in the test. The proposed approach could be a useful tool for the condition assessment of real bridge structures.

\section{ACKNOWLEDGEMENTS}

The work described in this paper was supported by the grants from the Hong Kong Research Grant Council Project No. PolyU5201/07E and from the Niche Area Project Funding of The Hong Kong Polytechnic University Project No. 1-BB6F. 


\section{References:}

[1] J.W. Lee, J.D. Kim, C.B. Yun, J.H. Yi, J.M. Shim, Health-monitoring method for bridges under ordinary traffic loadings, J. Sound. Vib. 257 (2002) 247-264.

[2] C.R. Farrar, G.H. James, System identification from ambient vibration measurements on a bridge, J. Sound. Vib. 205 (1999) 1-18.

[3] C.Y. Kim, D.S. Jung, N.S. Kim, S.D. Kwon, M.Q. Feng, Effect of vehicle weight on natural frequencies of bridges measured from traffic-induced vibration, Earthq. Eng. Eng. Vib. 2 (2003) 109-115.

[4] L. Majumder, C.S. Manohar, A time-domain approach for damage detection in beam structures using vibration data with a moving oscillator as an excitation source, J. Sound. Vib. 268 (2003) 699-716.

[5] T. Park, M. Noh, S. Lee, G.Z. Voyiadjis, Identification of a distribution of stiffness reduction in reinforced concrete slab bridges subjected to moving loads, J. Bridge. Eng. ASCE. 14 (2009) $355-365$.

[6] Q. Zhang, Ł. Jankowski, Z.D. Duan, Simultaneous identification of moving masses and structural damage, Struct. Multidiscip. O. 42 (2010) 907-922.

[7] F.T.K. Au, Y.S. Cheng, Y.K. Cheung, Vibration analysis of bridges under moving vehicles and trains: an overview, Prog. Struct. Eng. Mat. 3 (2001) 299-304.

[8] X.Q. Zhu, S.S. Law, Damage detection in simply supported concrete bridge structure under moving vehicular loads, J. Vib. Acoust. ASME. 129 (2007) 58-65.

[9] S.S. Law, J. Li, Updating the reliability of a concrete bridge structure based on condition assessment with uncertainties, Eng. Struct. 32 (2010) 286-296.

[10] D.E. Newland, An Introduction to Random Vibrations, Spectral and Wavelet Analysis, third ed., Longman Group Limited, England, 1993.

[11] A.M.R. Ribeiro, J.M.M. Silva, N.M.M. Maia, On the generalization of the transmissibility concept, Mech. Syst. Signal. Pr. 14 (2000) 29-35.

[12] S.S. Law, J. Li, Y. Ding, Structural response reconstruction with transmissibility concept in frequency domain, Mech. Syst. Signal. Pr. 25 (2011) 952-968.

[13] J. Li, S.S. Law, Substructural response reconstruction in wavelet domain, J. Appl. Mech. 
ASME. 78 (2011) 041010.

[14] K.F. Alvin, A.N. Robertson, G.W. Reich, K.C. Park, Structural system identification: from reality to models, Comput. Struct. 81 (2003) 1149-1176.

[15] S.S. Law, J.Q. Bu, X.Q. Zhu, S.L. Chan, Vehicle axle loads identification using finite element method, Eng. Struct. 26 (2004) 1143-1153.

[16] N.W. Newmark, A method of computation for structural dynamics, J. Eng. Mech. Div. ASCE. 85 (1959) 67-94.

[17] J.N. Juang, R.S. Pappa, An eigensystem realization algorithm for modal parameter identification and model reduction, J. Guid. Control. Dynam. 8 (1985) 583-592.

[18] A.N. Robertson, K.C. Park, K.F. Alvin, Extraction of impulse response data via wavelet transform for structural system identification, J. Vib. Acoust. ASME. 120 (1998) 252-260.

[19] S.S. Law, X.Y. Li, Wavelet-based sensitivity analysis of the impulse response function for damage detection, J. Appl. Mech. ASME. 74 (2007) 375-377.

[20] I. Daubechies, Ten Lectures on Wavelets, SIAM, Philadelphia, PA, 1992.

[21] R. Penrose, A generalized inverse for matrices, Math. Proc. Cambridge. 51 (1955) 406-413.

[22] Z.R. Lu, S.S. Law, Features of dynamic response sensitivity and its application in damage detection, J. Sound. Vib. 303 (2007) 305-329.

[23] S. Zivanovic, A. Pavic, P. Reynolds, Finite element modeling and updating of a lively footbridge: the complete process, J. Sound. Vib. 301 (2007) 126-145.

[24] K.W. Morton, D.F. Mayers, Numerical Solution of Partial Differential Equations, An Introduction, second ed., Cambridge University Press, 2005.

[25] X.Y. Li, S.S. Law, Adaptive Tikhonov regularization for damage detection based on nonlinear model updating, Mech. Syst. Signal. Pr. 24(2010) 1646-1664.

[26] Y.W. Kwon, H.C. Bang, The Finite Element Method using MATLAB, second ed., USA: CRC Press LLC, 2000.

[27] S.S. Law, J.Q. Bu, X.Q. Zhu, S.L. Chan, Moving load identification on a simply supported orthotropic plate, Int. J. Mech. Sci. 49 (2007) 1262-1275.

[28] J.J. Wu, Use of moving distributed mass element for the dynamic analysis of a flat plate undergoing a moving distributed load, Int. J. Numer. Meth. Eng. 71 (2007) 347-362.

[29] AASHTO LRFD bridge design specifications. American Association of State Highway and 
Transportation Offices. Washington, DC. 2007.

[30] X.Q. Zhu, S.S. Law, Dynamic load on continuous multi-lane bridge deck from moving vehicles, J. Sound. Vib. 251 (2002): 697-716.

[31] S.S. Law, J. Li, S.Q. Wu, Prestress identification in box-girder bridge deck under traffic load, Proceedings of the $3^{\text {rd }}$ International Symposium on Environment Vibrations: Prediction, Monitoring, Mitigation and Evaluation (ISEV 2007), Taipei, TAIWAN, 2007, 631-638.

[32] ISO 8606: 1995(E). Mechanical vibration - road surface profiles - reporting of measured data.

[33] A. Furukawa, H. Otsuka, J. Kiyono, Structural damage detection method using uncertainty frequency response functions, Comput-Aided Civ. Inf. 21 (2006) 292-305. 
Table 1 - Sensor placement configuration of Example 1

\begin{tabular}{cc}
\hline Sensor Placement Configuration & Sensor locations \\
\hline First-set & Node 14(z), 51(z) \\
Second-set & Node 8(z), 21(z), 45(z), 56(z) \\
\hline
\end{tabular}

Note: "Node 14(z)" denotes that the sensor is placed along the $z$-direction at Node 14.

Table 2 - Information on convergence of Example 1

\begin{tabular}{ccc}
\hline & No noise & $10 \%$ noise \\
\hline Required iterations & 5 & 18 \\
Error of convergence & $5.39 \times 10^{-5}$ & $9.56 \times 10^{-5}$ \\
\hline
\end{tabular}

Table 3 - Sensor placement configuration of Example 2

\begin{tabular}{cc}
\hline Sensor Placement Configuration & Sensor locations \\
\hline First-set & Node 8(z), 20(z), 21(z), 45(z), 56(z) \\
Second-set & Node 14(z), 51(z) \\
\hline
\end{tabular}

Table 4 - Information on convergence of Example 2

\begin{tabular}{ccc}
\hline & No noise & $10 \%$ noise \\
\hline Required iterations & 6 & 22 \\
Error of convergence & $2.01 \times 10^{-5}$ & $9.85 \times 10^{-5}$ \\
\hline
\end{tabular}


Table 5- Damage scenarios with initial model errors

\begin{tabular}{cccc}
\hline Scenario & Damage & Model errors & Noise effect \\
\hline 1 & & $5 \%$ random stiffness changes & $10 \%$ \\
& $20 \%$ stiffness reductions & in all elements & \\
\cline { 3 - 4 } & in both $28^{\text {th }}$ and $29^{\text {th }}$ elements & $10 \%$ increase in the & $10 \%$ \\
\cline { 3 - 4 } & & support stiffness & \\
\cline { 3 - 4 } & & Include both types of model errors & $10 \%$ \\
\hline
\end{tabular}

Table 6 - Identified frequencies and damping ratios in different states

\begin{tabular}{ccccccc}
\hline $\begin{array}{c}\text { Structural } \\
\text { State }\end{array}$ & \multicolumn{2}{c}{ Without prestress } & With prestress (initial state) & \multicolumn{2}{c}{ Cracked (Damaged state) } \\
\hline Mode & $\begin{array}{c}\text { Frequency } \\
(\mathrm{Hz})\end{array}$ & Damping & Frequency & Damping & Frequency & Damping \\
& 31.998 & 0.016 & 33.386 & 0.016 & 31.817 & 0.011 \\
\hline 1 & 82.757 & 0.0014 & 97.411 & 0.028 & 90.719 & 0.029 \\
\hline
\end{tabular}

Table 7 - Sensor placement configuration of experimental study

\begin{tabular}{cc}
\hline Sensor Placement Configuration & Sensor locations \\
\hline First-set & S1, S3, S5, S4, S6 \\
Second-set & S2, S7 \\
\hline
\end{tabular}


Table 8 - Observed crack locations and heights

\begin{tabular}{ccccccccccccc}
\hline $\begin{array}{c}\text { Distance from } \\
\text { left support of } \\
\text { beam (mm) }\end{array}$ & 700 & 1000 & 1100 & 1160 & 1440 & 1620 & 1800 & 1950 & 2125 & 2260 & 2340 & 2540 \\
\hline $\begin{array}{c}\text { Web Element } \\
\text { No. }\end{array}$ & 4 & 5 & 5 & 5 & 6 & 7 & 8 & 8 & 9 & 9 & 9 & 10 \\
$\begin{array}{c}\text { Crack Height } \\
\text { (mm) }\end{array}$ & 226 & 156 & 191 & 300 & 270 & 305 & 280 & 248 & 273 & 286 & 243 & 244 \\
\hline $\begin{array}{c}\text { Distance from } \\
\text { left support of } \\
\text { beam (mm) }\end{array}$ & 2680 & 2800 & 2900 & 3030 & 3130 & 3220 & 3330 & 3510 & 3680 & 3850 & 4000 & 4100 \\
\hline $\begin{array}{c}\text { Web Element } \\
\text { No. }\end{array}$ & 10 & 11 & 11 & 12 & 12 & 12 & 13 & 13 & 14 & 14 & 14 & 15 \\
$\begin{array}{c}\text { Crack Height } \\
\text { (mm) }\end{array}$ & 261 & 251 & 120 & 260 & 118 & 274 & 220 & 220 & 192 & 158 & 140 & 126 \\
\hline
\end{tabular}



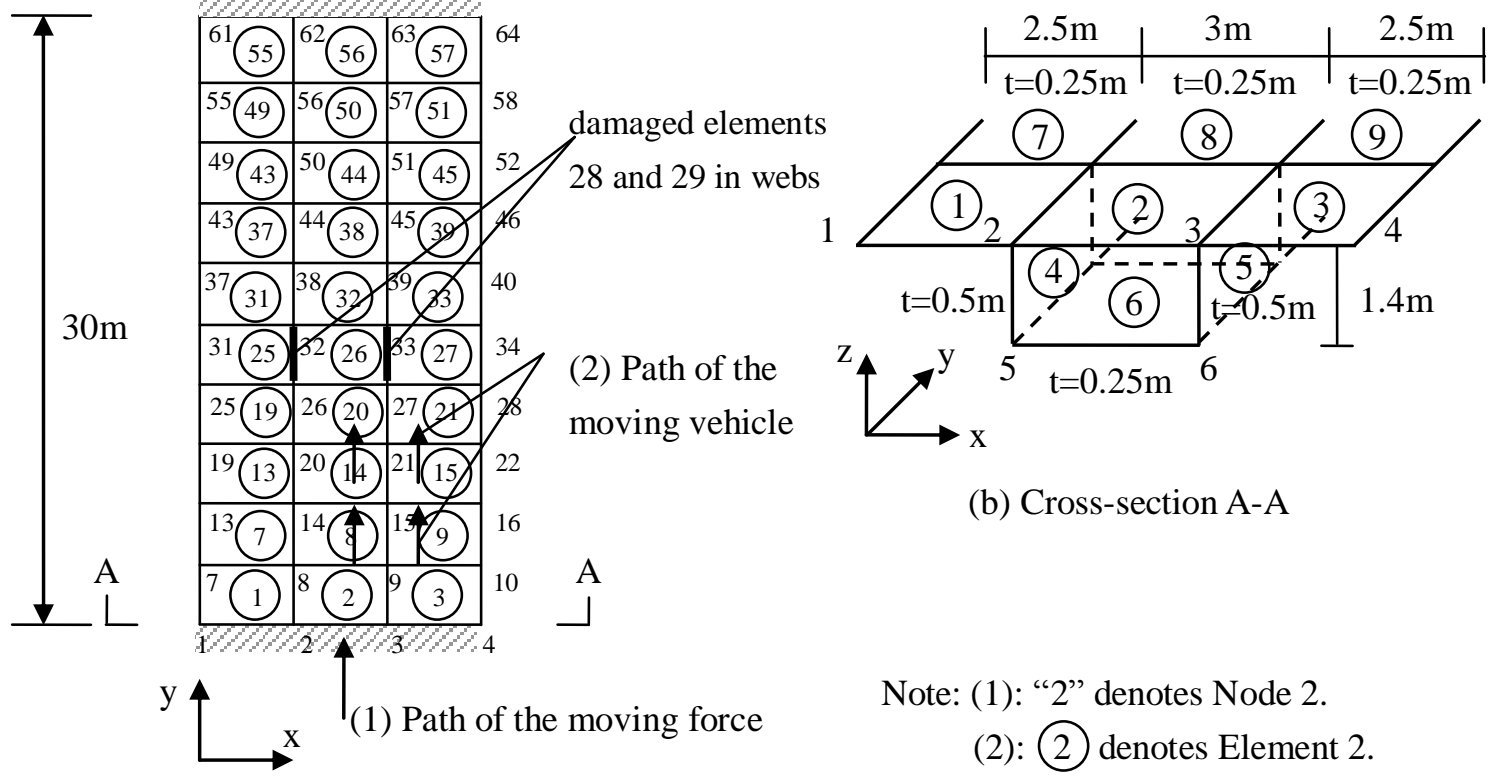

(a) Plan view of the box-section girder

Figure 1 - Finite element model of the box-section girder structure 


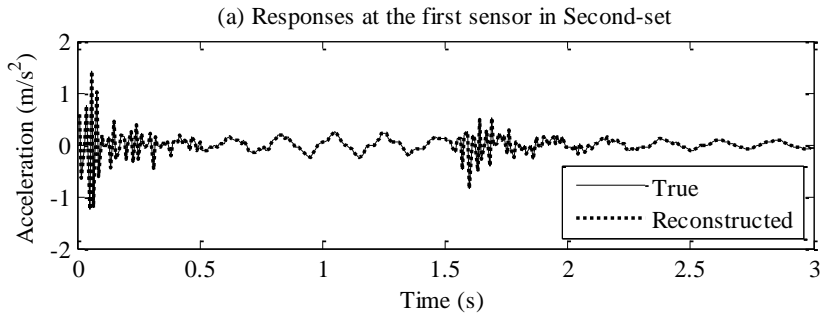

(c) Responses at the second sensor in Second-set

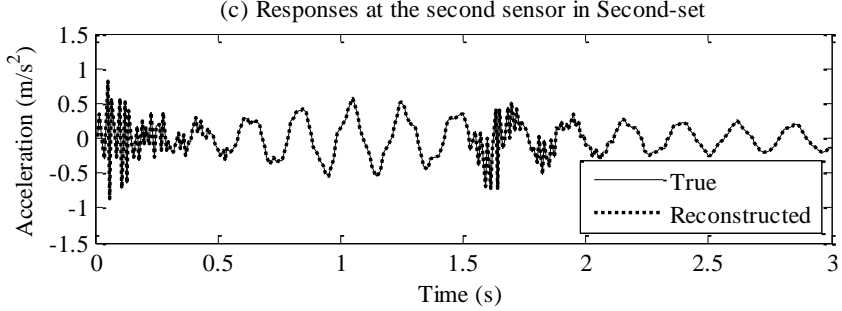

(e) Responses at the third sensor in Second-set

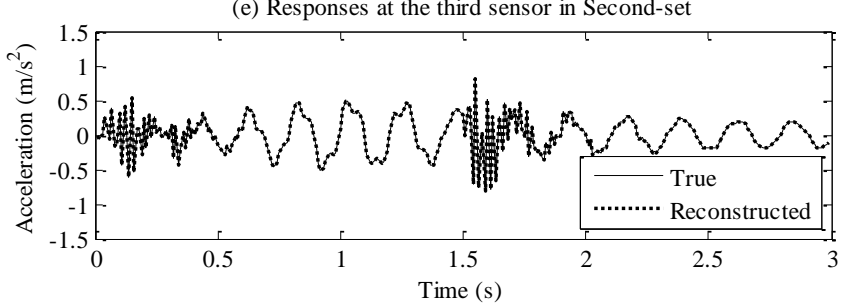

(g) Responses at the fourth sensor in Second-set

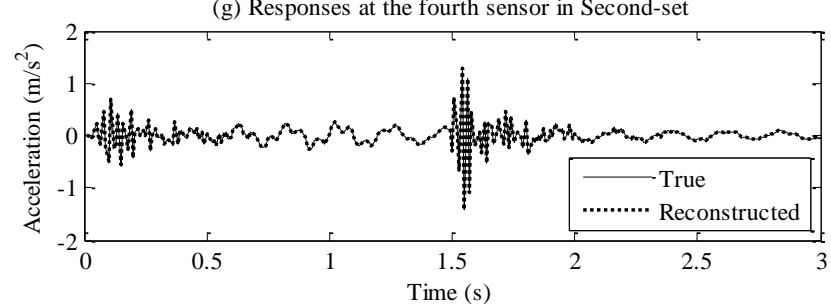

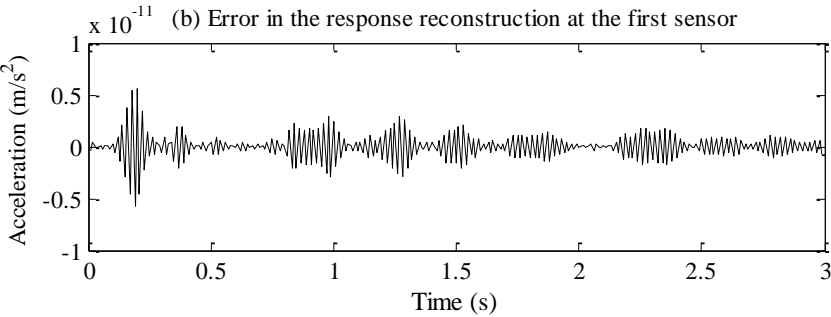
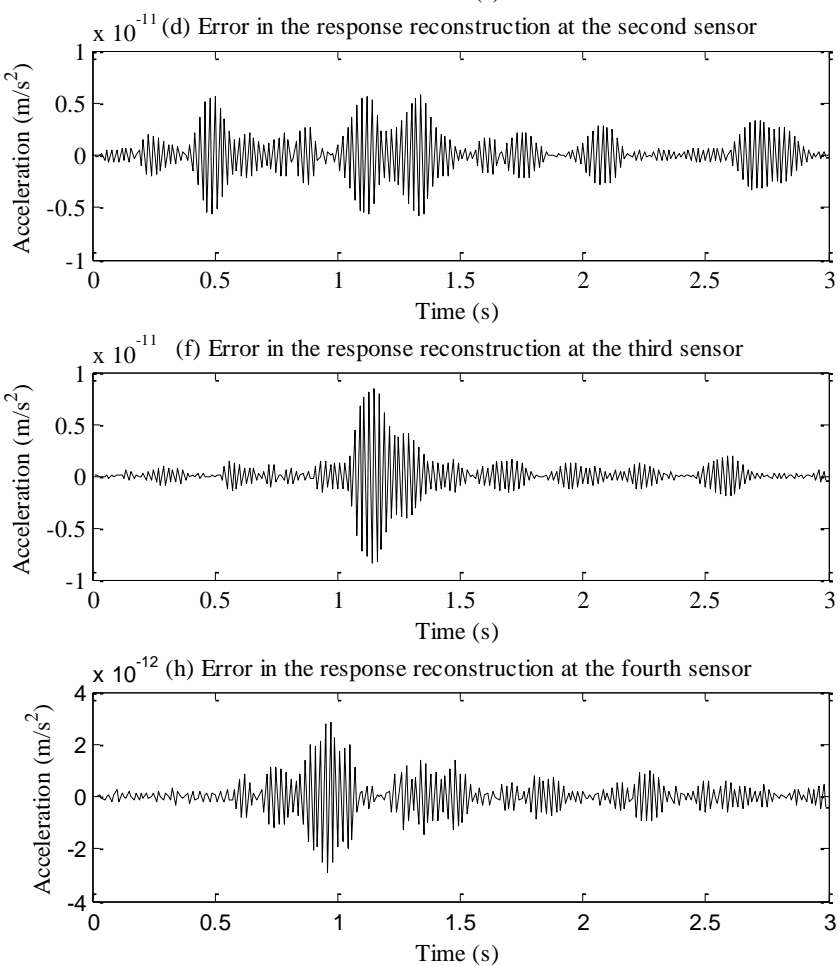

Figure 2 - True and reconstructed responses in the Second-set response vector of Example 1 


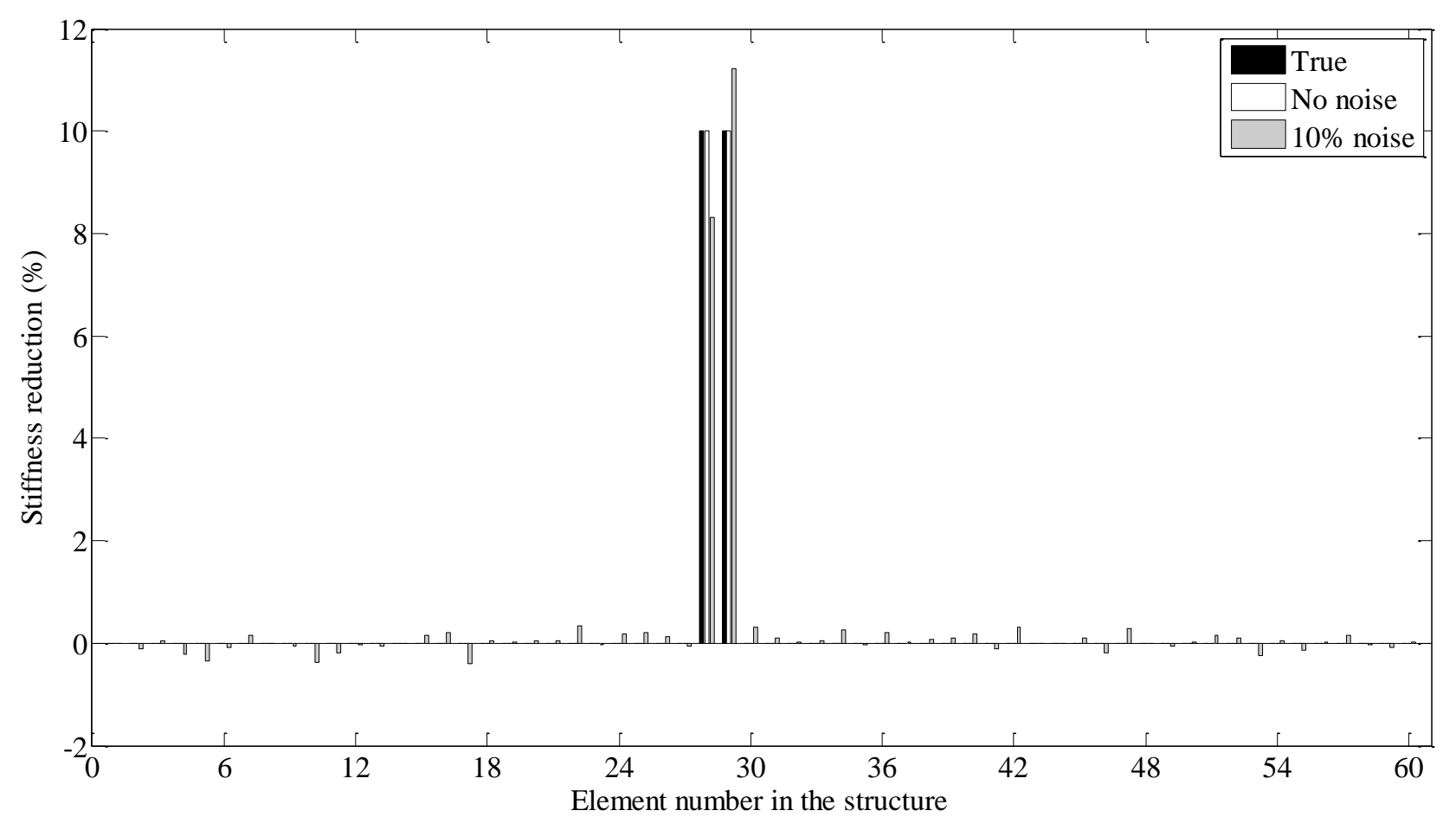

Figure 3 - Damage identification results of Example 1 

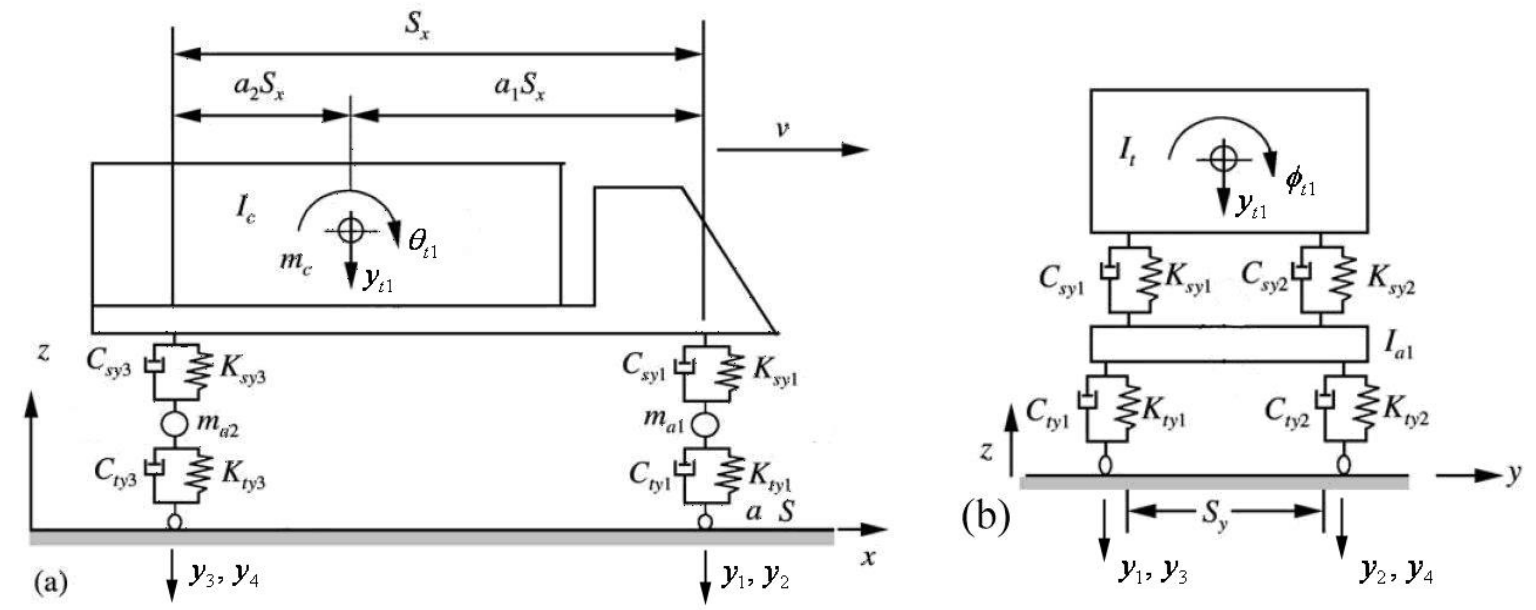

Figure 4 - A two-axle three-dimensional vehicle with seven DOFs 
(a) Responses at the first sensor in Second-set

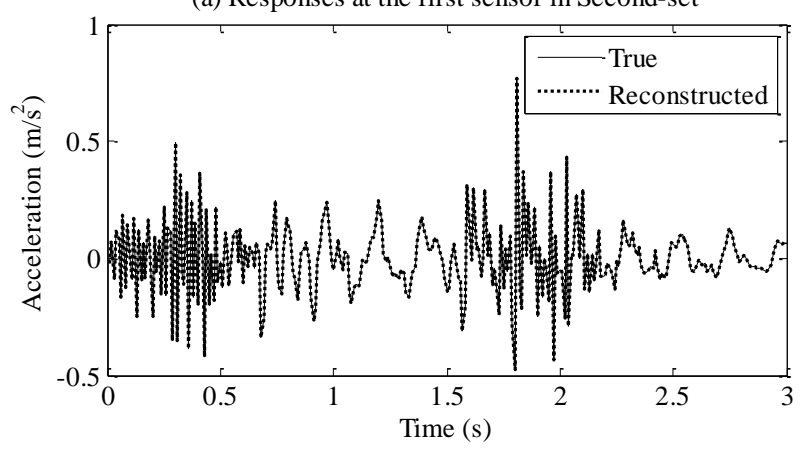

(c) Responses at the second sensor in Second-set

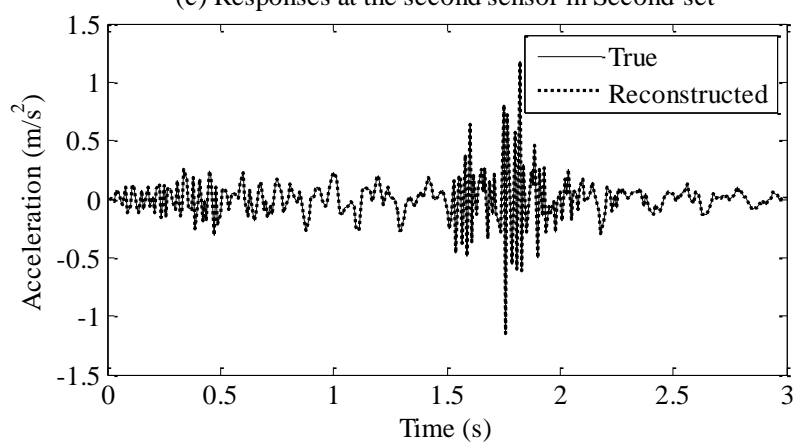

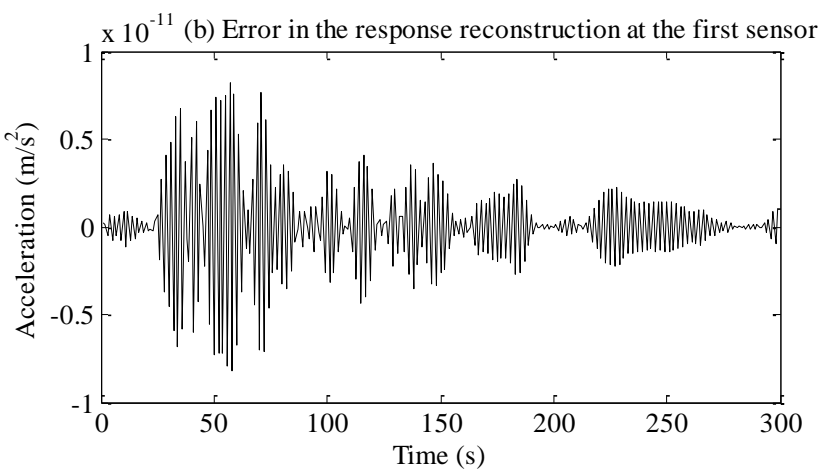

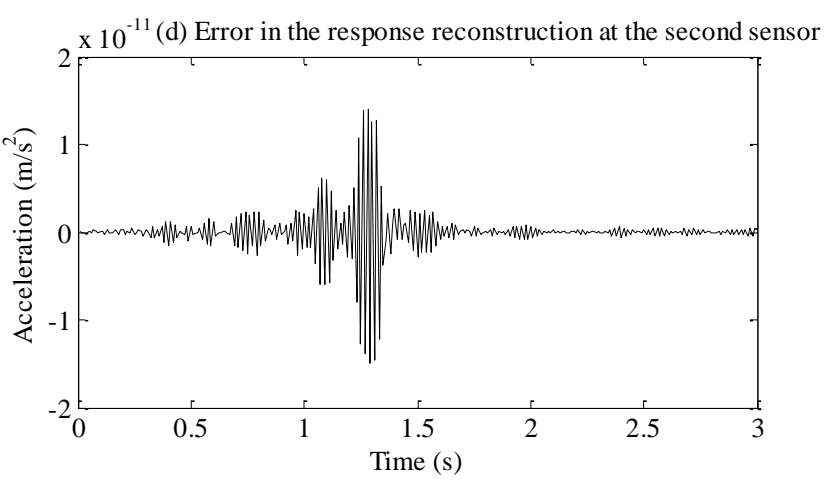

Figure 5 - True and reconstructed responses in the Second-set response vector of Example 2 


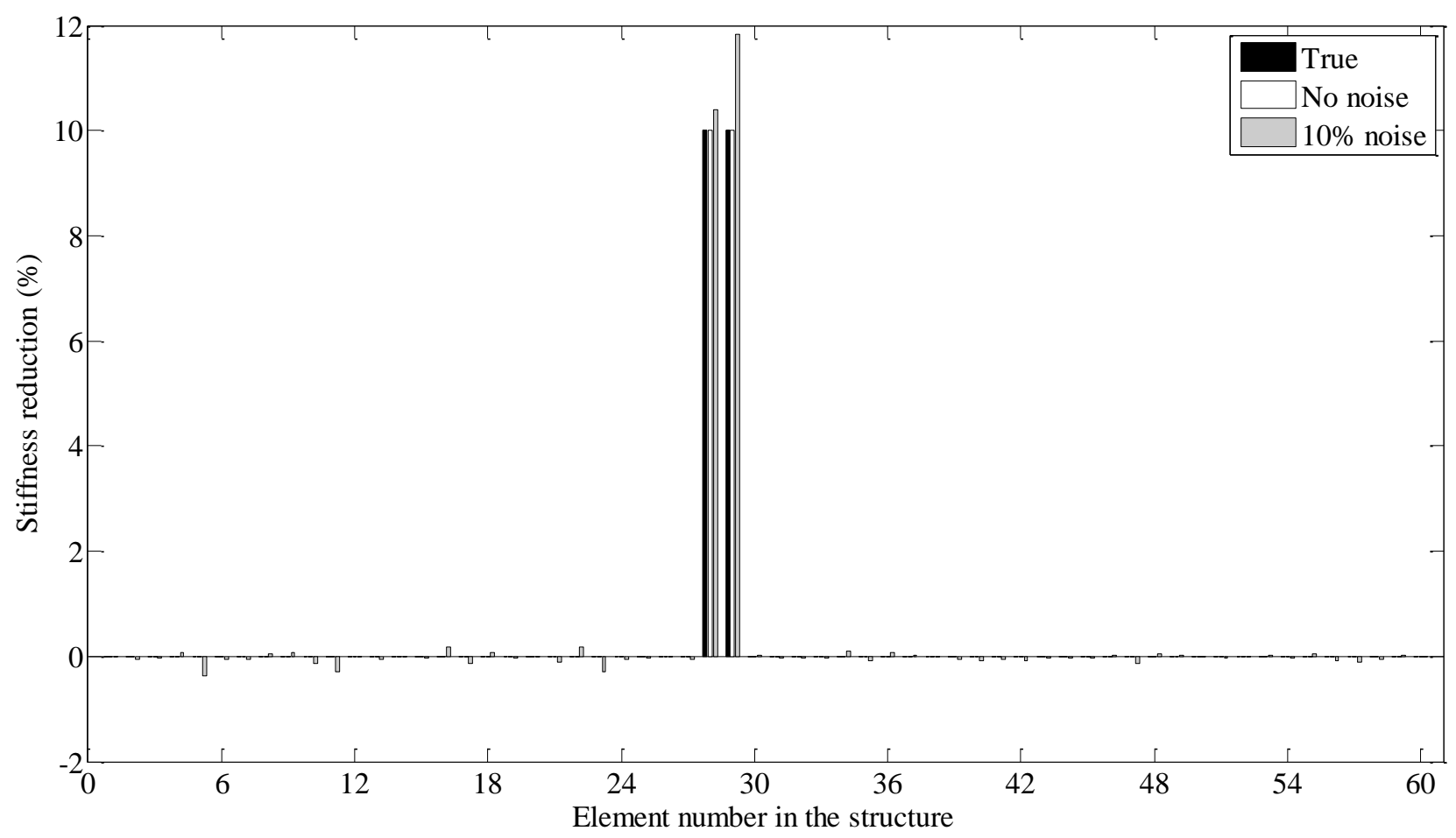

Figure 6 - Damage identification results of Example 2 
(a) Scenario 1

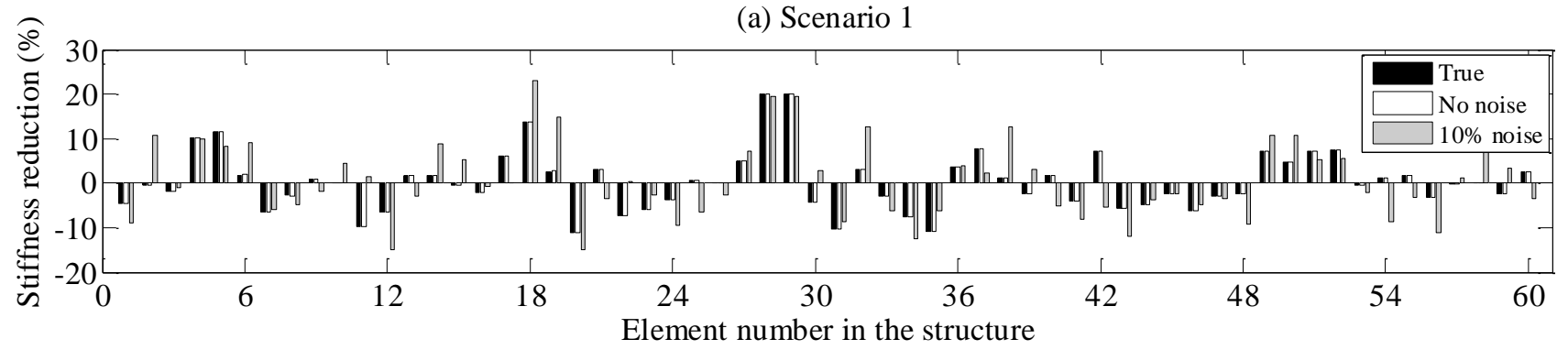

(b) Scenario 2

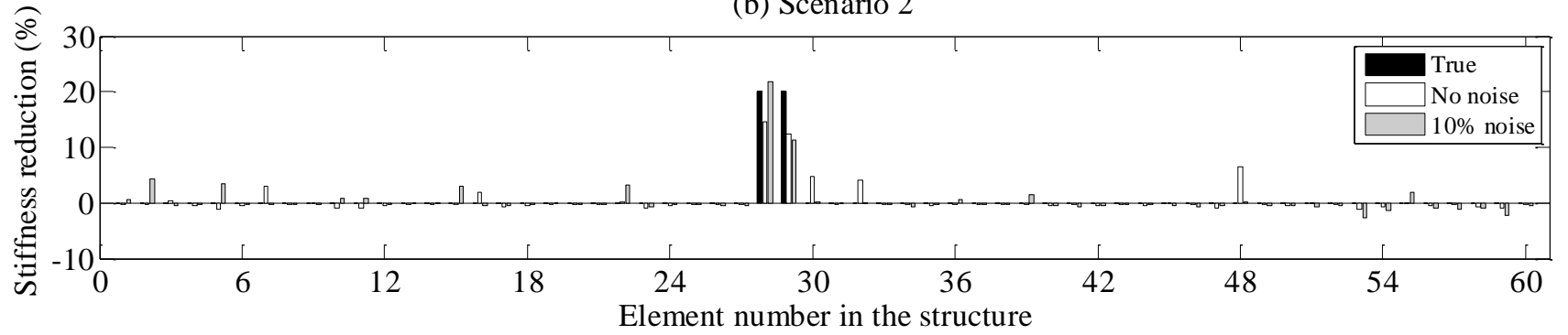

(c) Scenario 3

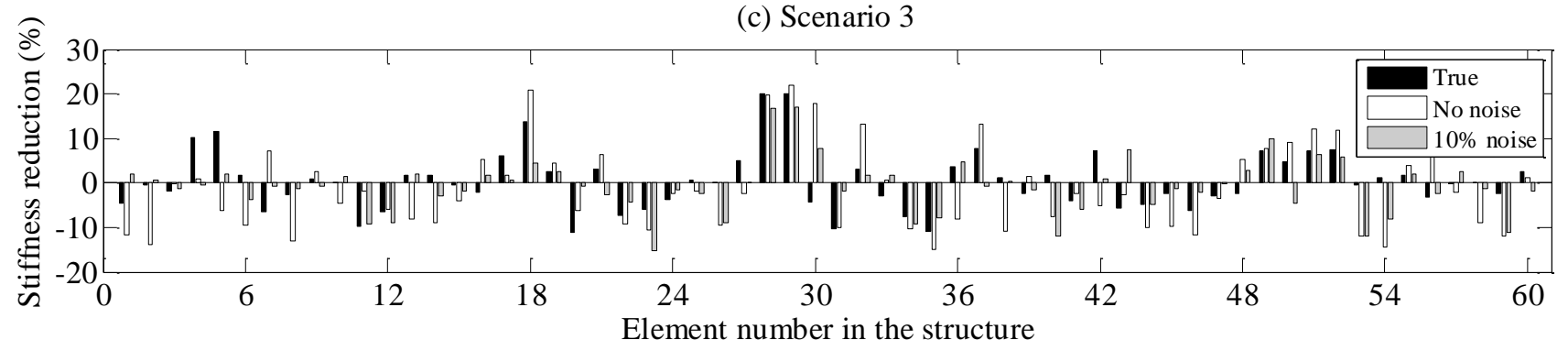

Figure 7- Damage identification results with initial model errors 


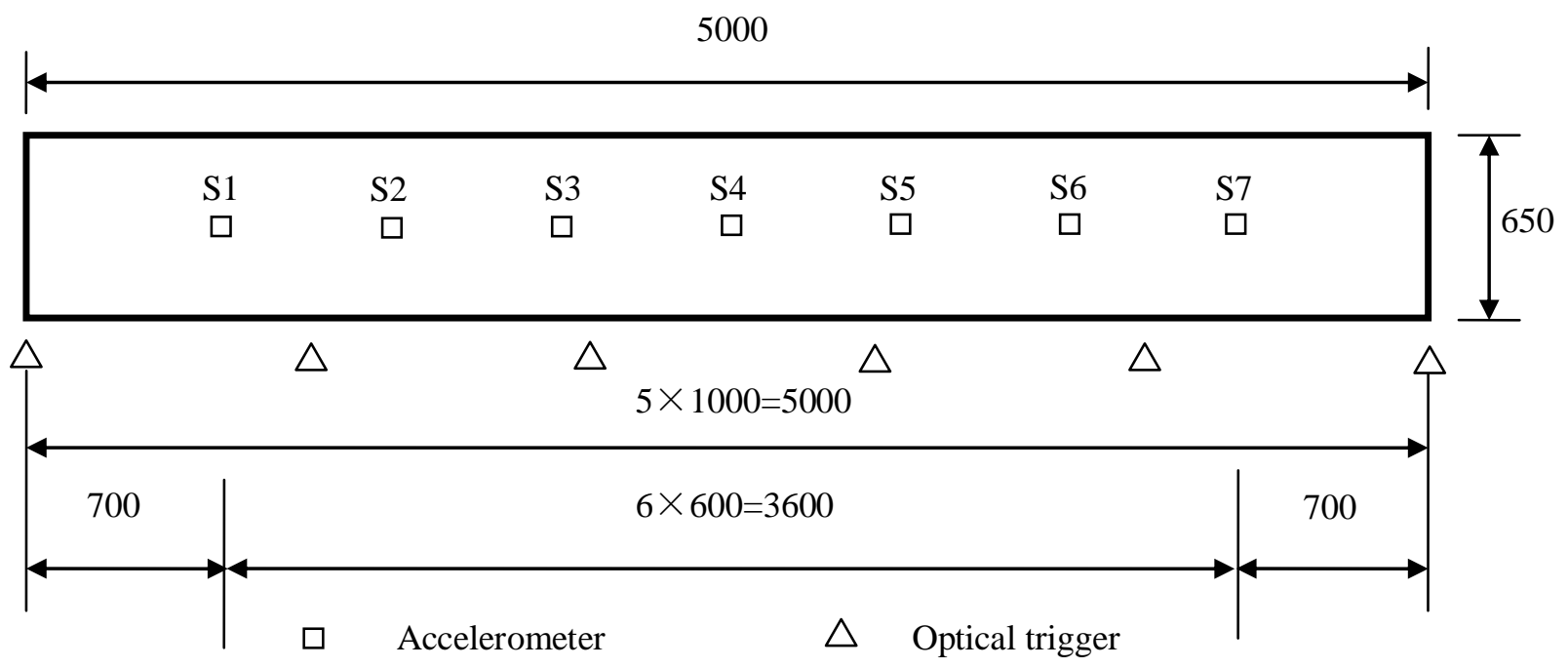

(a) Plan view

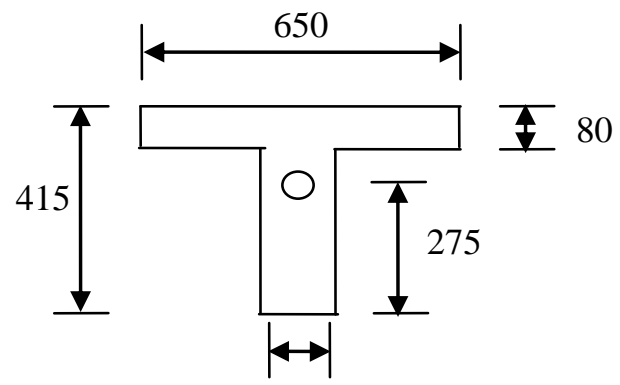

150

$\bigcirc$ Prestress location at ends

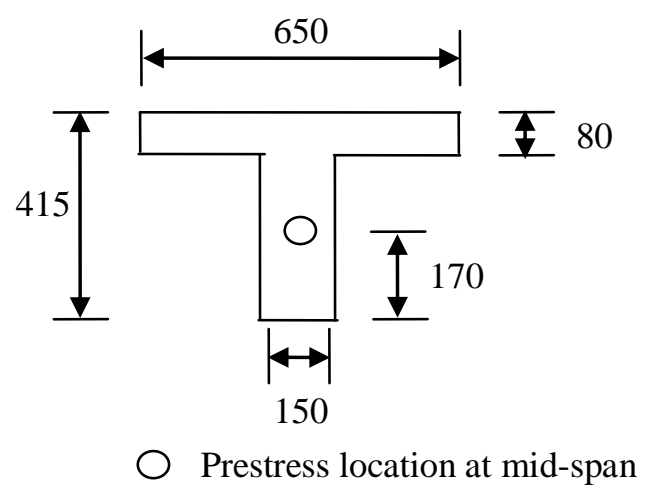

(b) Cross-sections

Figure 8 - The prestressed concrete beam (Unit: $\mathrm{mm}$ ) 


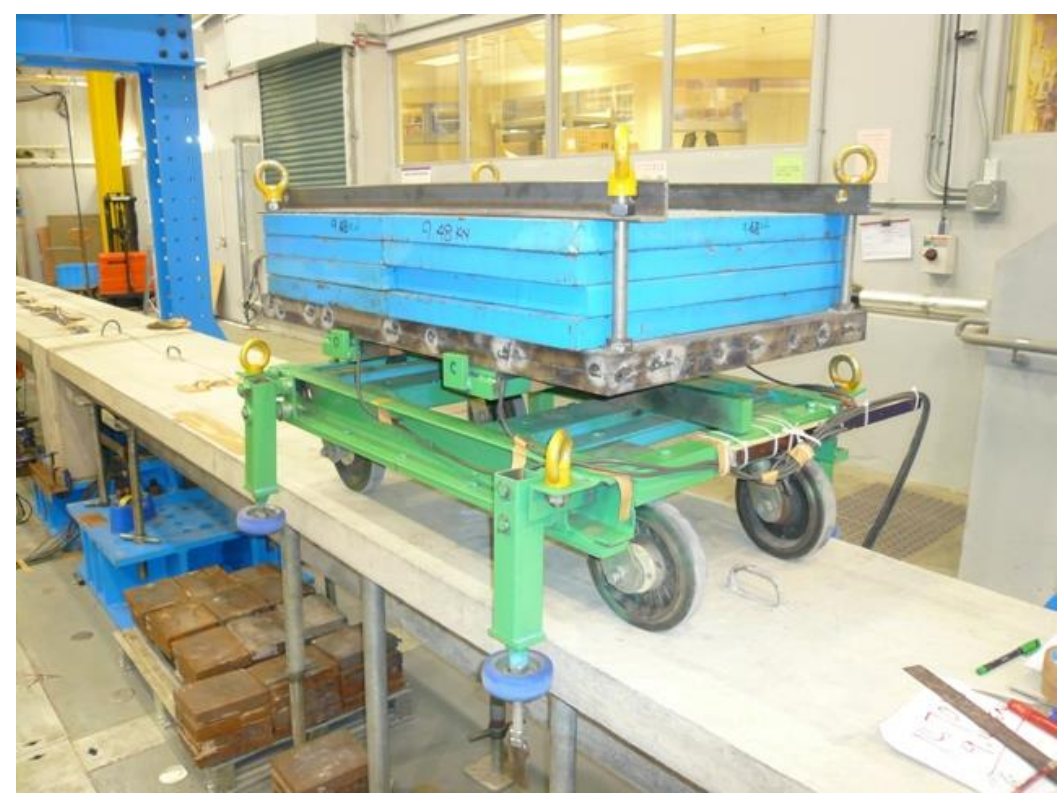

(a) Vehicle

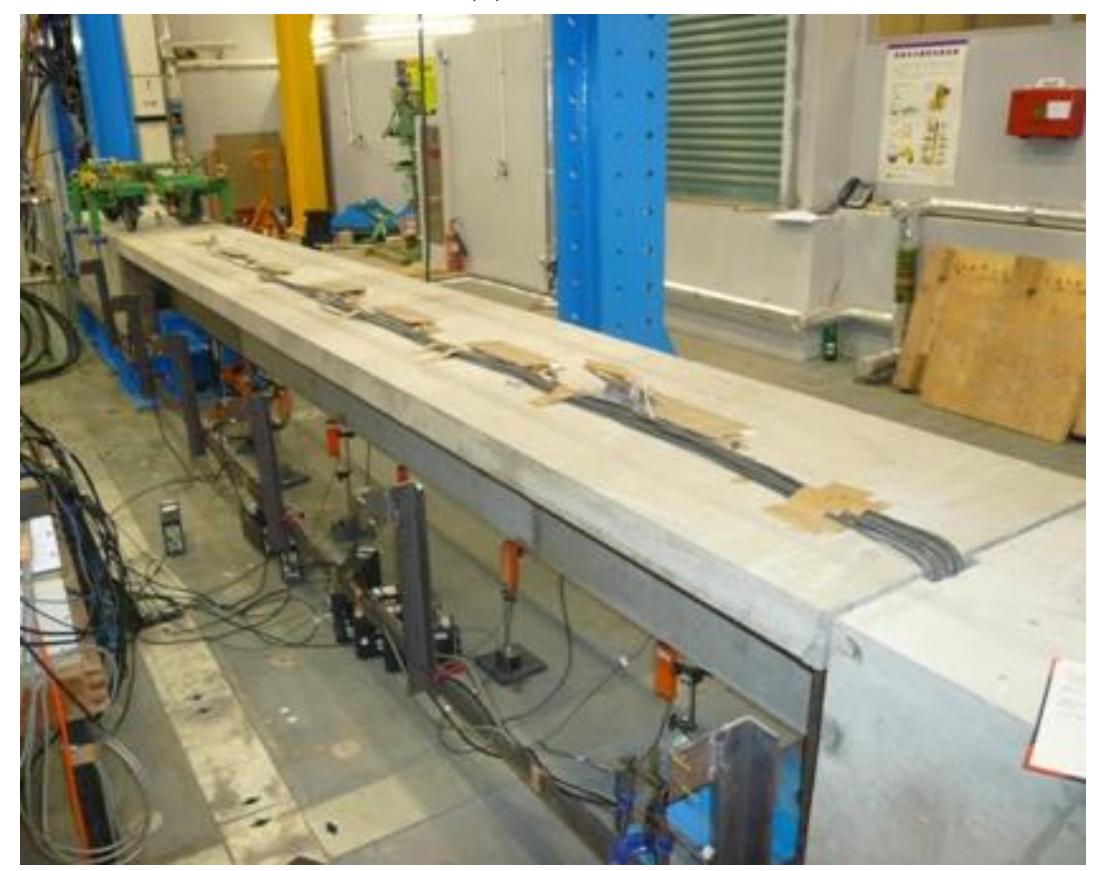

(b) Optical triggers and accelerometers

Figure 9 - The vehicle model, optical triggers and acceleration sensors 

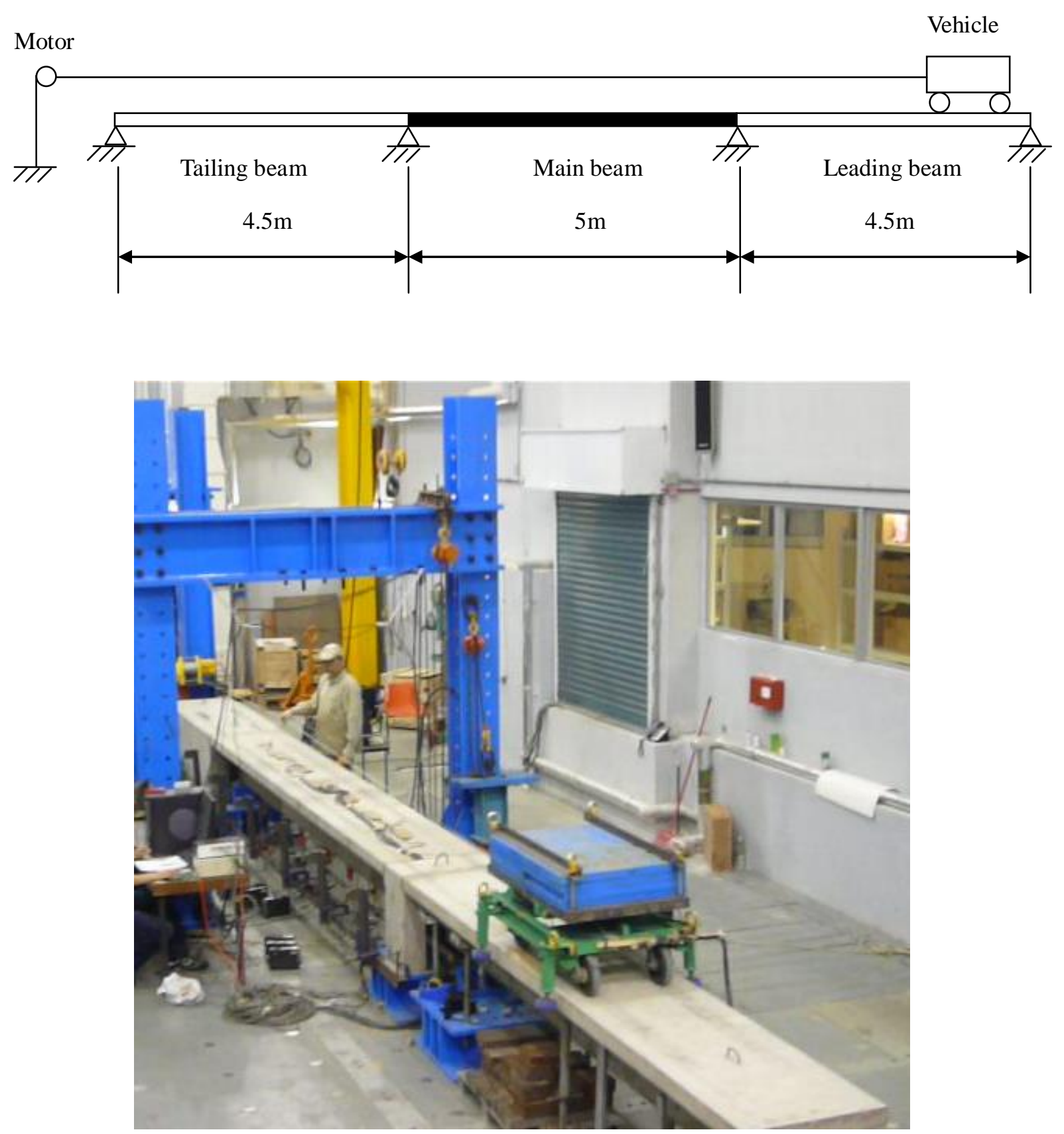

Figure 10 - Schematic experimental setup 


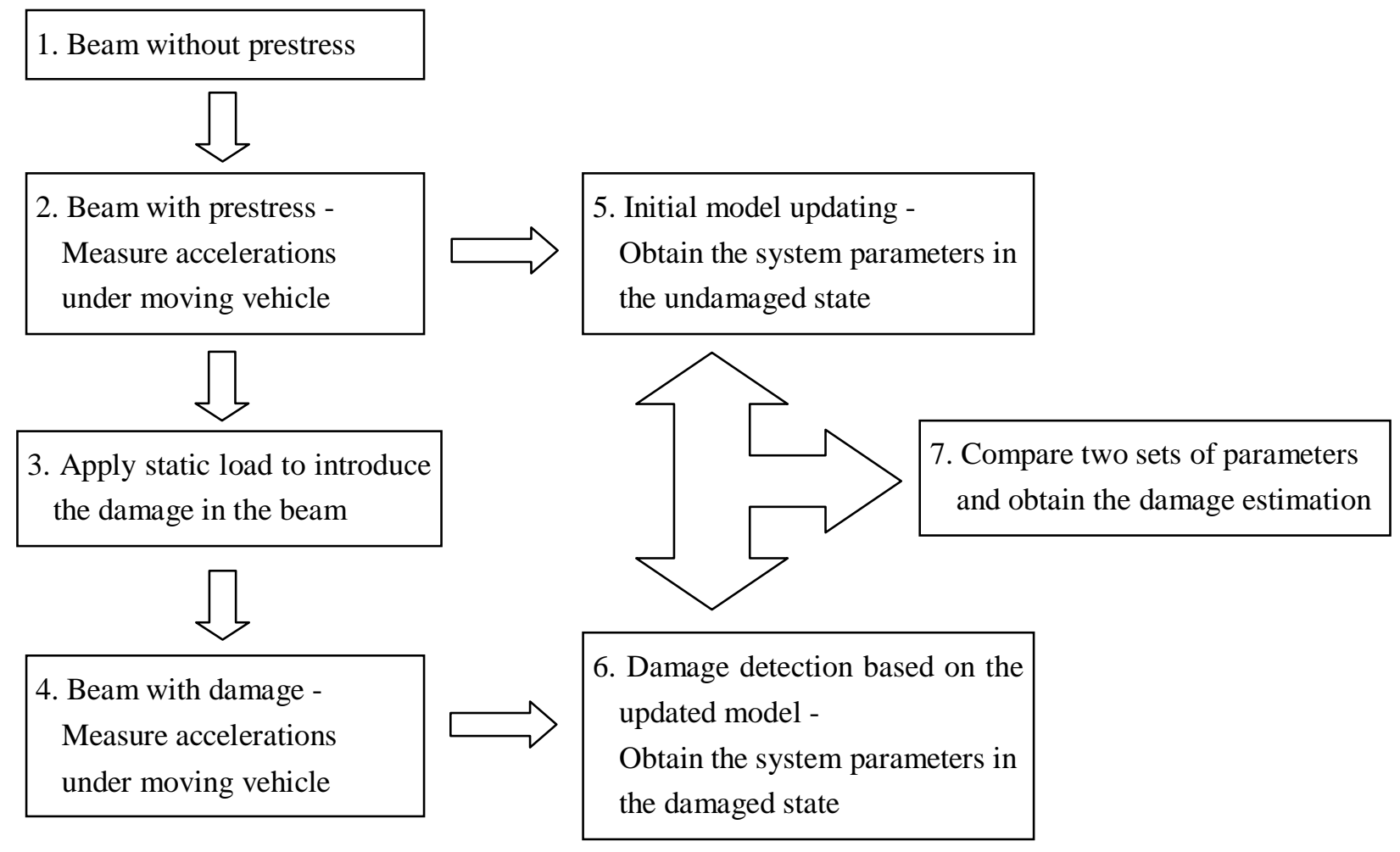

Figure 11 - Flow chart of the experimental damage identification 


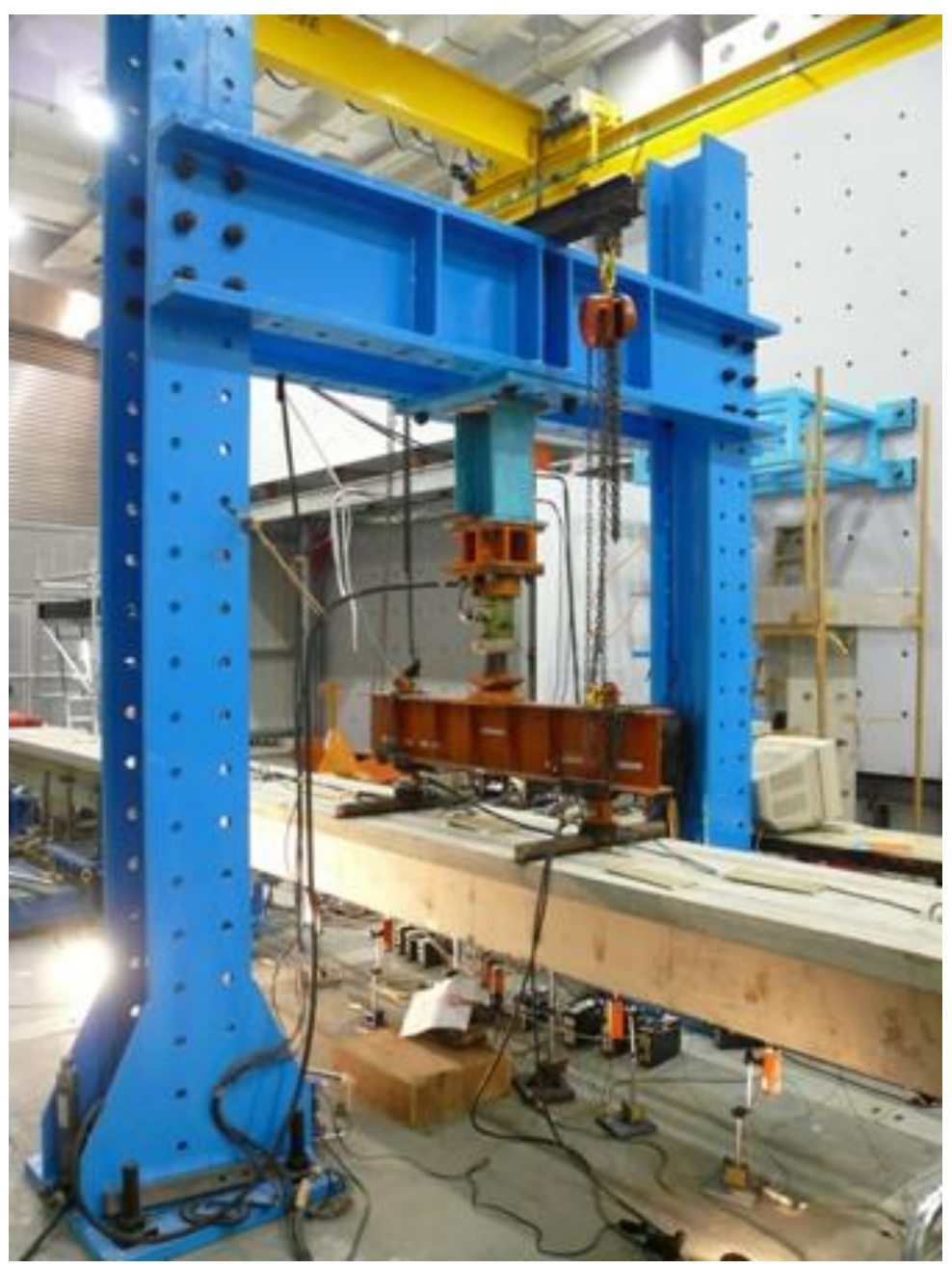

Figure 12 - Application of static load to the beam 


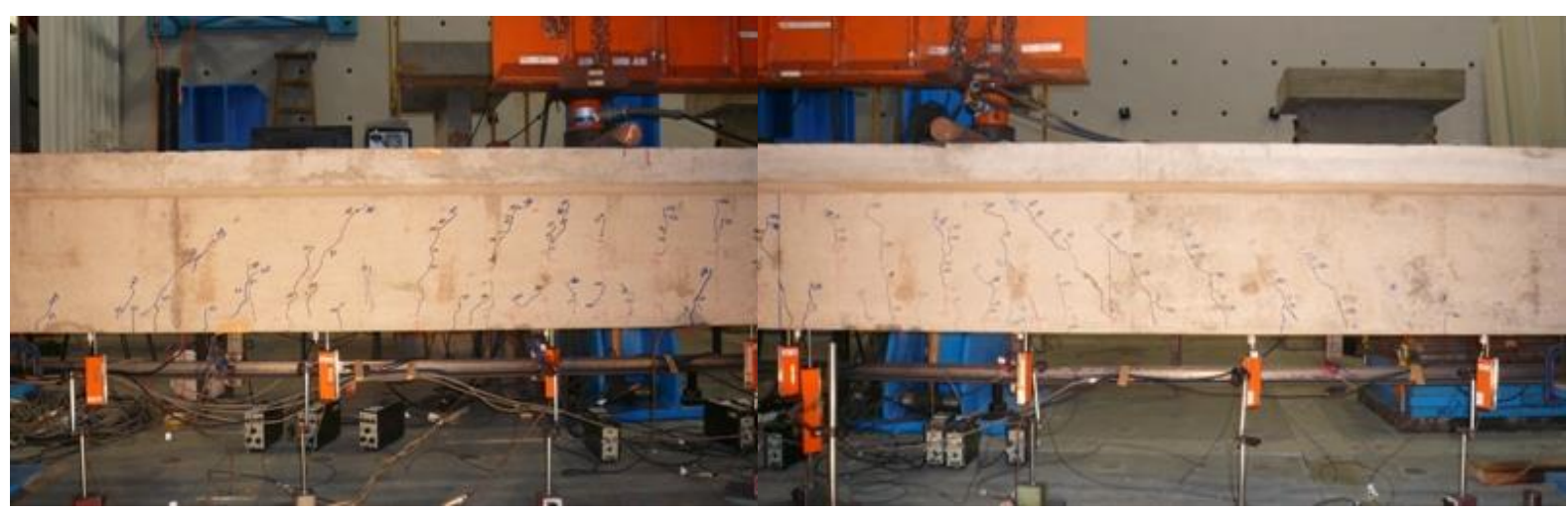

Figure 13 - Observed crack pattern in web elements of the beam 


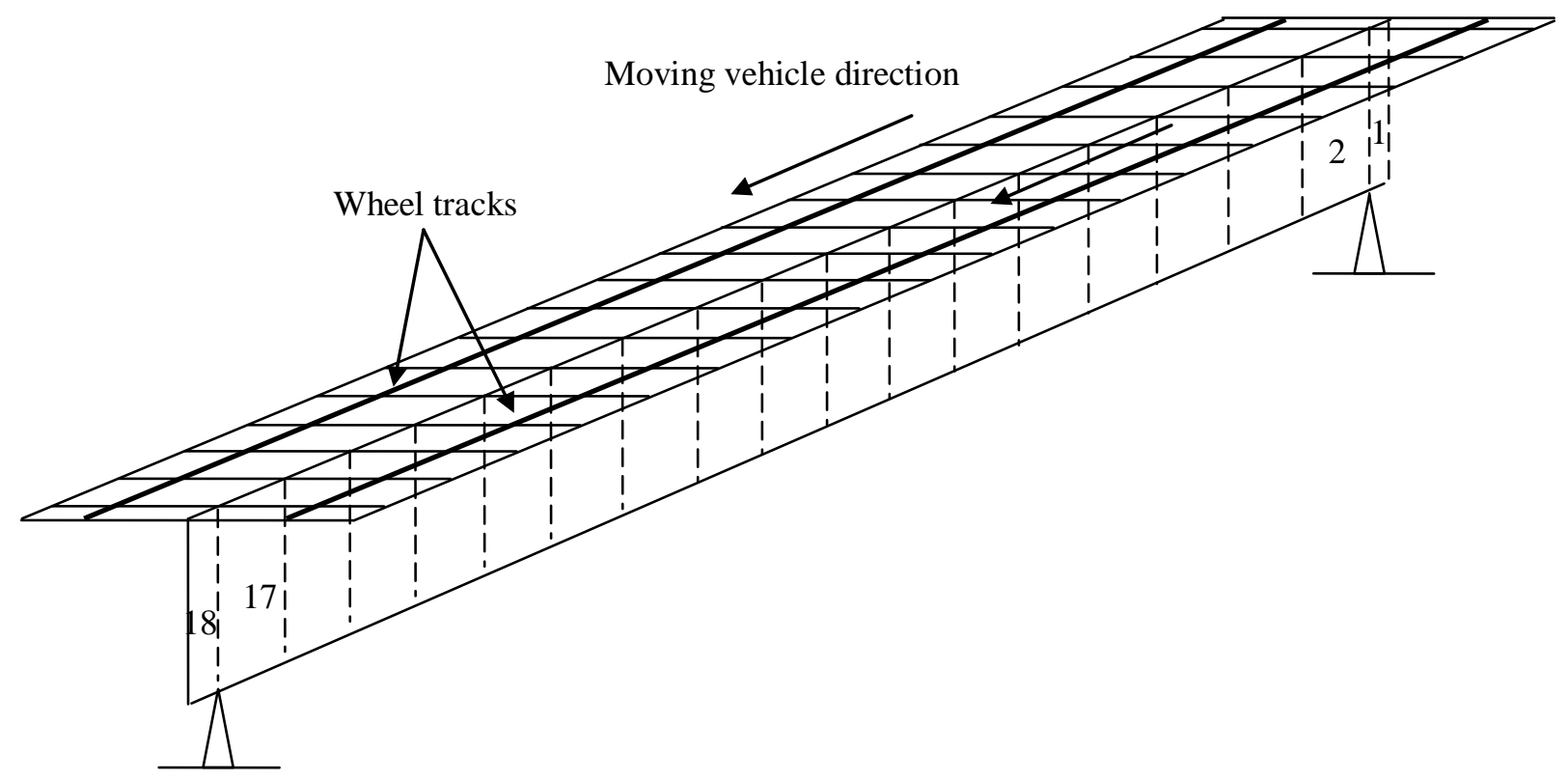

Moving vehicle direction

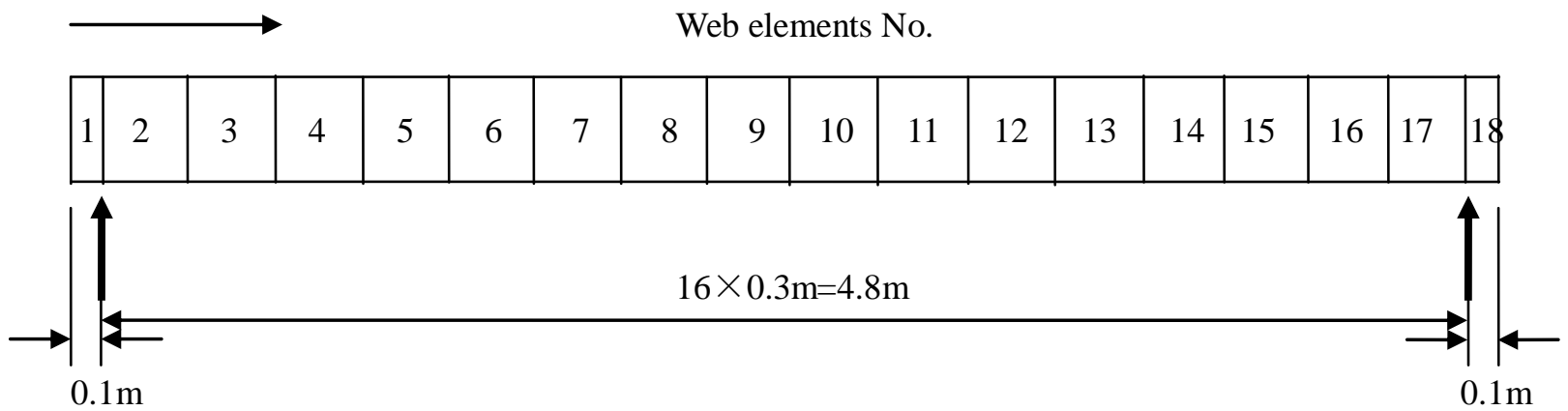

Figure 14 - Finite element model of the beam and web element numbers 


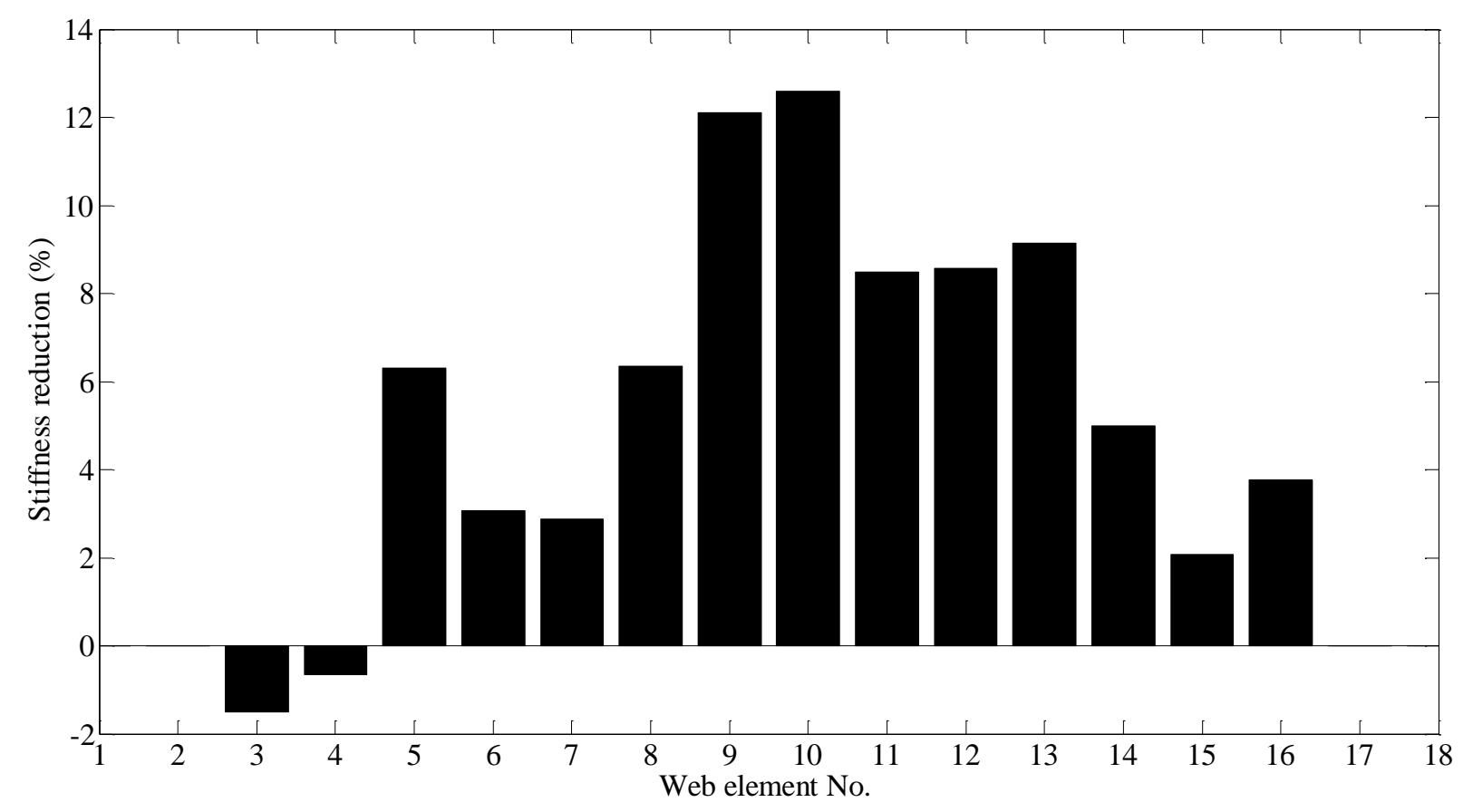

Figure 15 - Damage identification results in the web elements of the beam 